EPJ Web of Conferences 7, 02005 (2010)

DOI:10.1051/epjconf/20100702005

(C) Owned by the authors, published by EDP Sciences, 2010

\title{
Quark-Hadron Mixed Phase
}

V.D. Toneev ${ }^{1, a}$

JINT, Dubna, 141980 Russia

Abstract. The concept of a mixed quark-hadron phase is considered. Dynamics of heavy-ion collisins in a large energy range is outlined. Various possible probes of the mixed phase manifestation as well as modern status of experimental signals are discussed.

I am grateful to my colleagues A.Khvorostukhin, V.Skokov and

A.Shanenko for fruitful collaboration.

a e-mail: toneev@theor.jinr.ru

This is an Open Access article distributed under the terms of the Creative Commons Attribution-Noncommercial License 3.0, which permits unrestricted use, distribution, and reproduction in any noncommercial medium, provided the original work is properly cited. 


\title{
Quark-Hadron Mixed Phase
}

\author{
V. Toneev
}

Bogoliubov Laboratory of Theoretical Physics

- Introductory remarks

- Mixed-phase concept

- A glance at collision dynamics

- Status of signals for a phase transition

- Concluding remarks

\section{Introductory remarks}

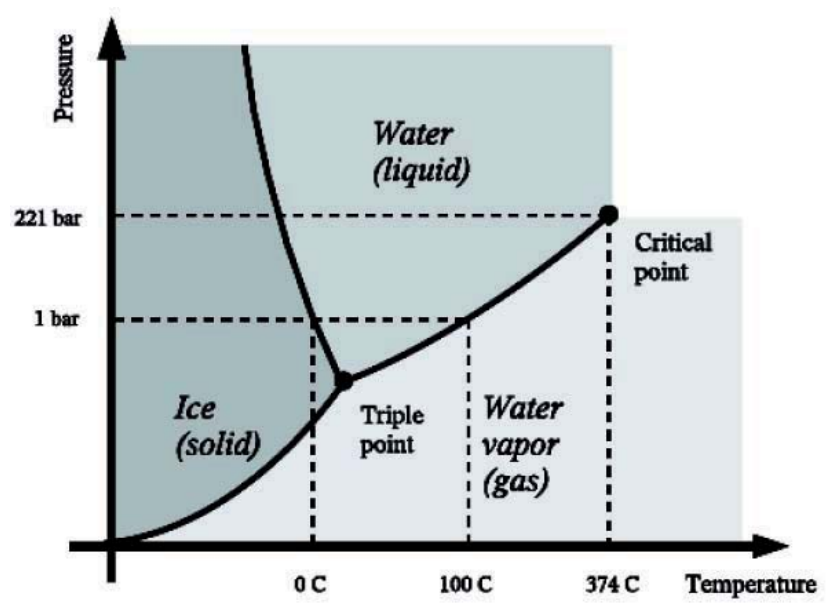




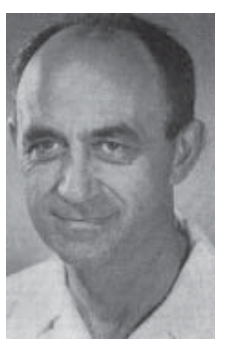

\section{Phase diagrams}

E.Fermi: Notes on themodymamics and statistics, 1953
G.Baym: Nucl. Phys. A418 (1984) 433c

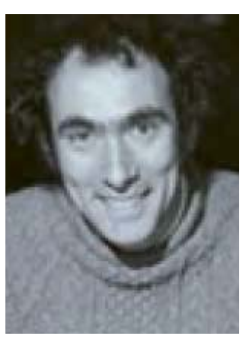

PHASE DIAGRAM OF NUCLEAR MATTER

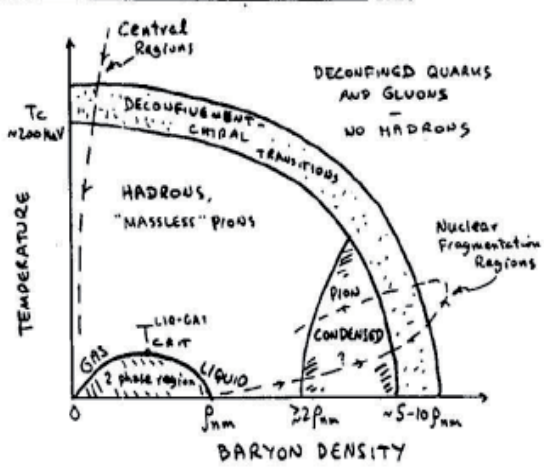

Nuclear matter

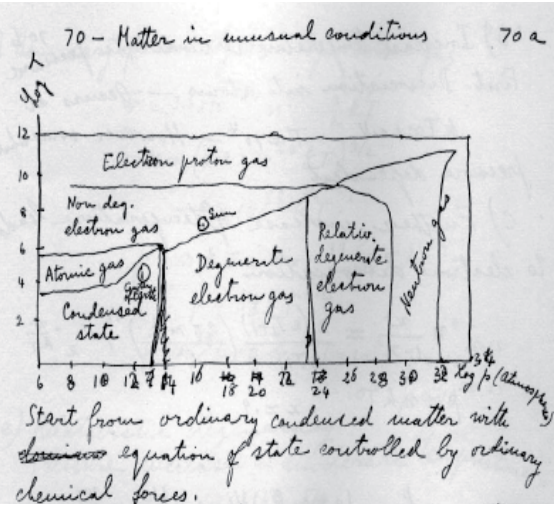

clemical fones.

Phase diagram - artist's view

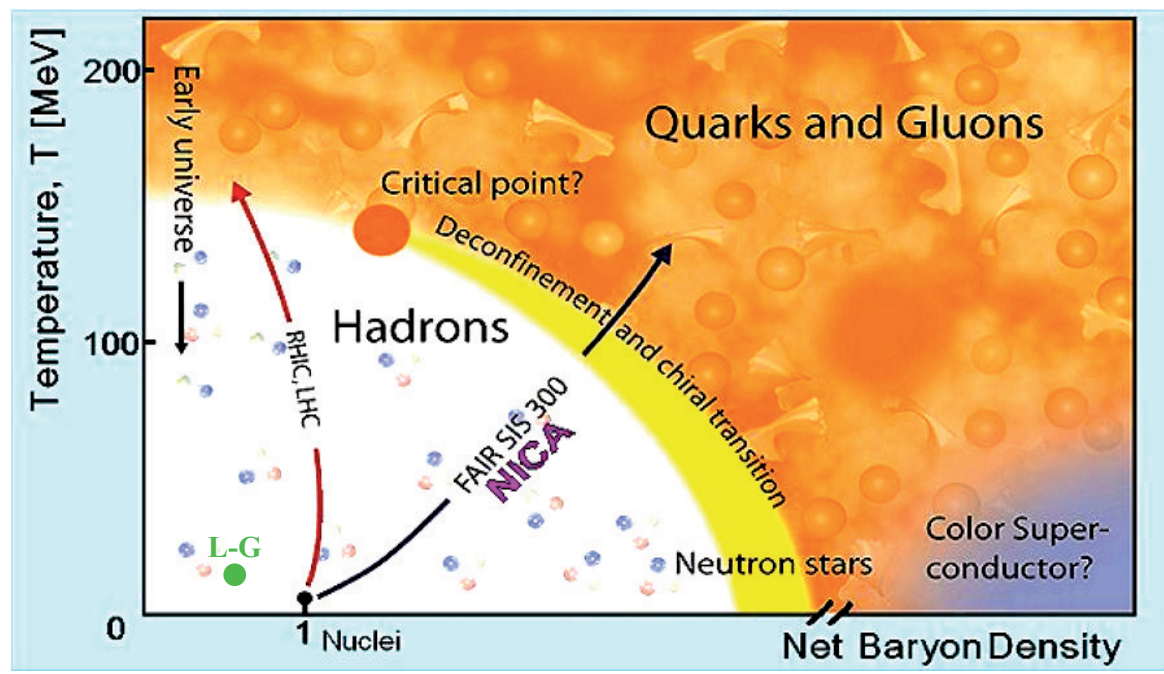

Phases of strongly interacting nuclear matter 


\section{Phase diagram for QCD matter (scales)}

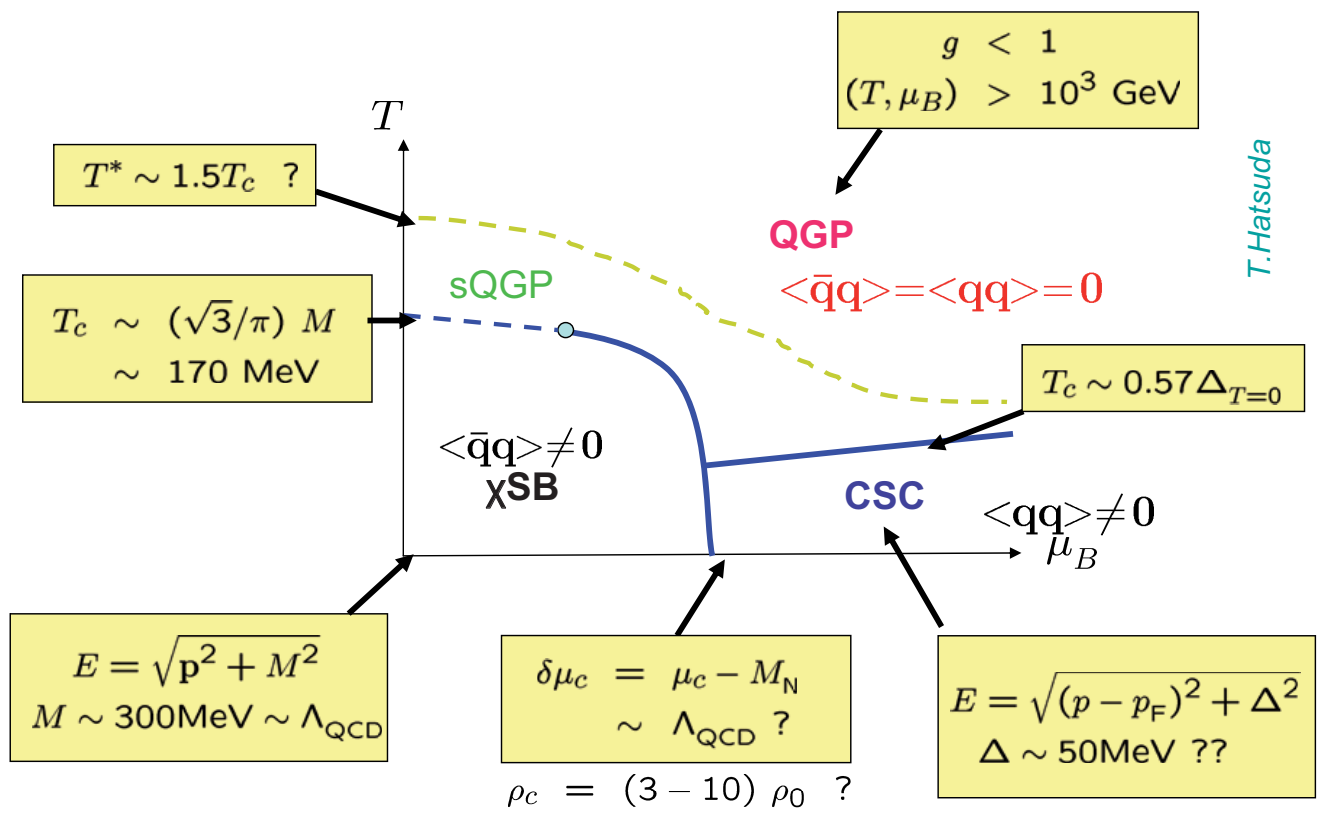

Mixed-phase concept 


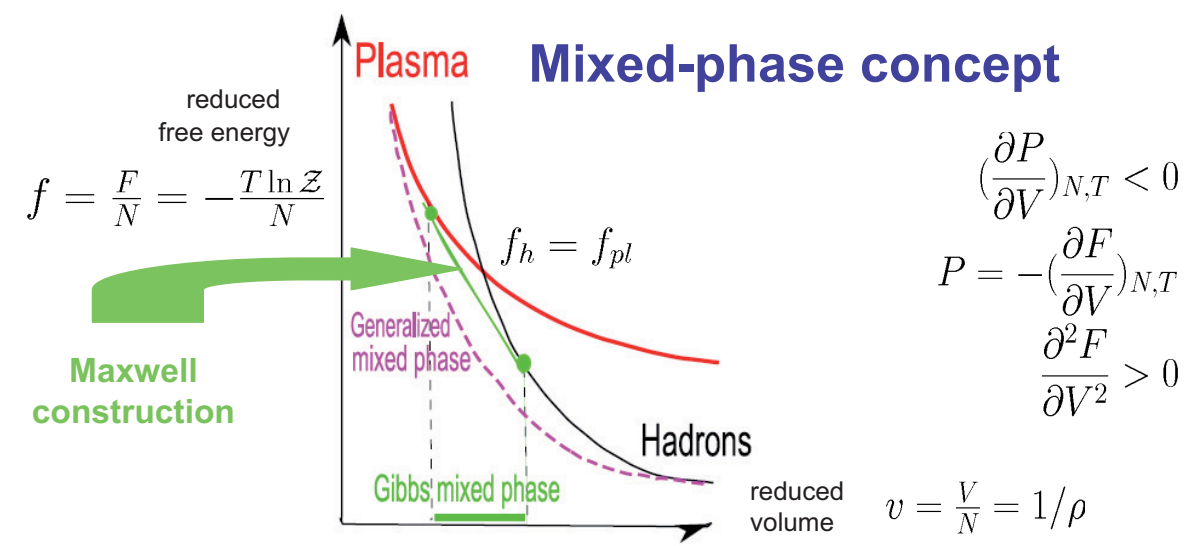

The Gibbs mixed phase (spatially separated)

Generalized mixed phase (homogeneous)
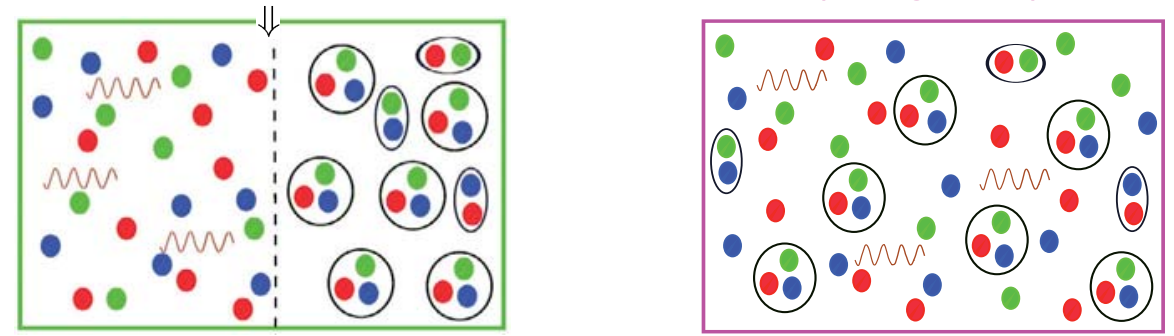

\section{Deconfinement transition (an illustrative example)}

$$
\begin{aligned}
& P=g_{i}\left(\frac{\pi^{2}}{90}\right) T^{4} \\
& \text { for the massless } \\
& \text { Stefan-Boltzmann gas } \\
& \varepsilon=g_{i}\left(\frac{\pi^{2}}{30}\right) T^{4}=3 P \\
& \mathrm{~g}_{\mathrm{i}} \text { - degeneracy factor } \\
& c_{s}^{2}=P / \varepsilon=1 / 3
\end{aligned}
$$

Two-phase model: pions $\Leftrightarrow$ quarks+gluons (pl)

$$
\begin{aligned}
& g_{\pi}=3 \\
& \begin{array}{l}
\text { Pion } \\
\text { gas }
\end{array} \\
& g_{p l}=2\left(\frac{N_{c} N_{f}}{4}+N_{c}^{2}-1\right) \\
& P_{p l}=37\left(\frac{\pi^{2}}{90}\right) T^{4}-B \\
& N_{f}=2, N_{c}=3 \\
& \varepsilon_{\pi}=3\left(\frac{\pi^{2}}{90}\right) T^{4}=3 P_{\pi} \\
& \varepsilon_{p l}=37\left(\frac{\pi^{2}}{30}\right) T^{4}+B=3 P_{p l}+4 B
\end{aligned}
$$

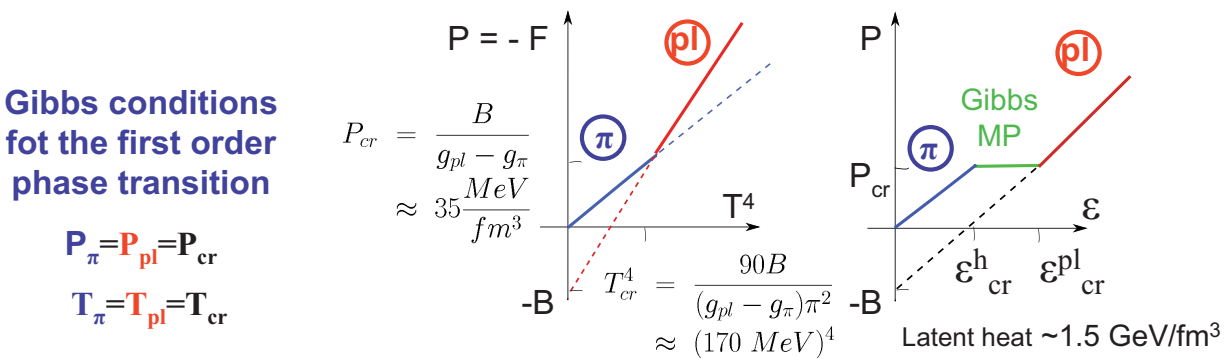




\section{Simplest example (no conserved charge)}

Entropy density : $\sigma(\varepsilon)$

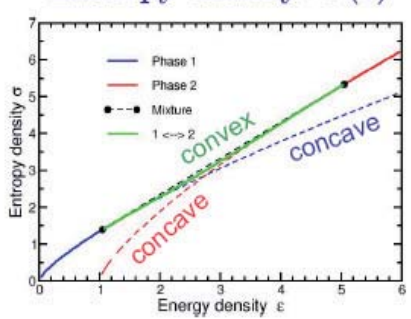

Inverse temperature: $\beta(\varepsilon)=\partial \sigma / \partial \varepsilon$

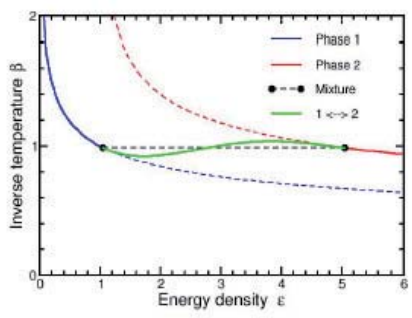

$\checkmark$ Equation of State

Pressure: $p(\varepsilon)=T \sigma-\varepsilon$

Pressure: $p(T)$
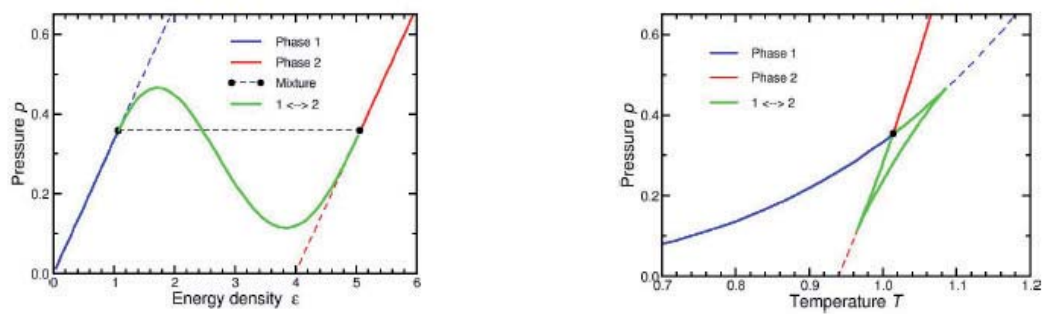

J.Randrup, Nucl.Phys. A752 (2005) 384c

\section{Familiar example (one conserved charge)}

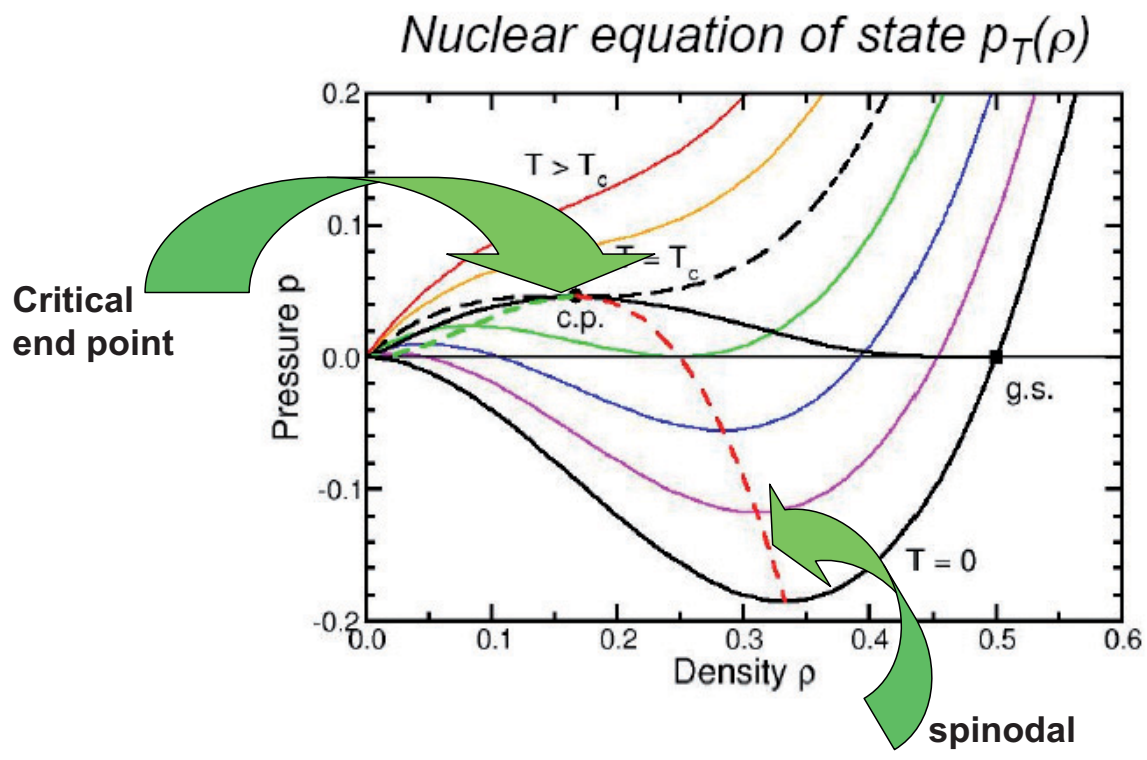




\section{Nuclear phase diagram in different representation (one conserved charge)}
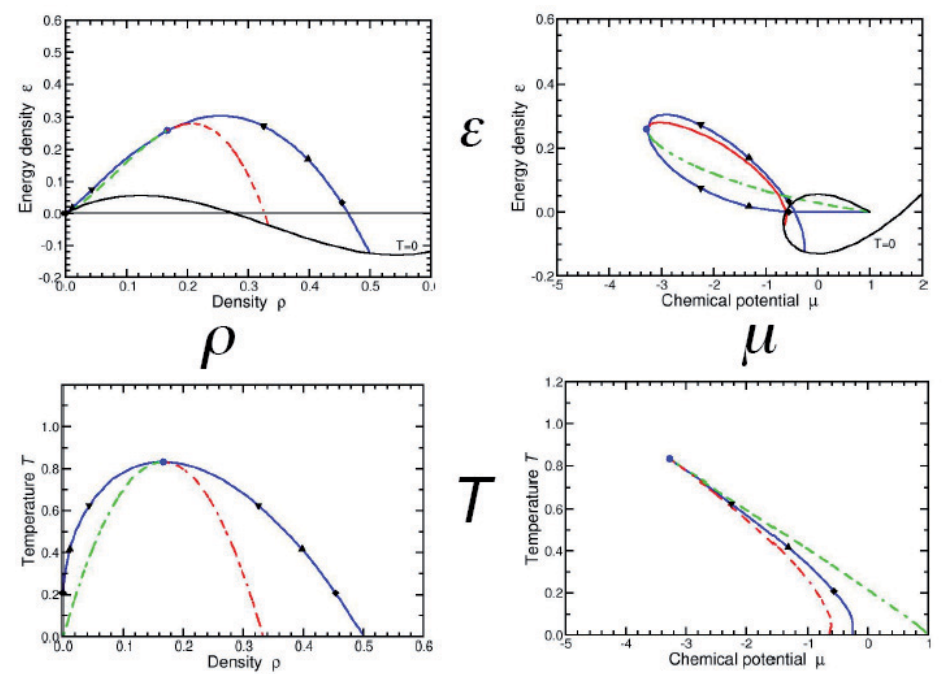

J.Randrup, CEOD, Darmstadt, July 9-13, 2007

\section{Two conserved charges}

for an extensive thermodynamic quantity $A=\lambda A_{2}+(1-\lambda) A_{1} \quad \lambda=V_{2} / V$
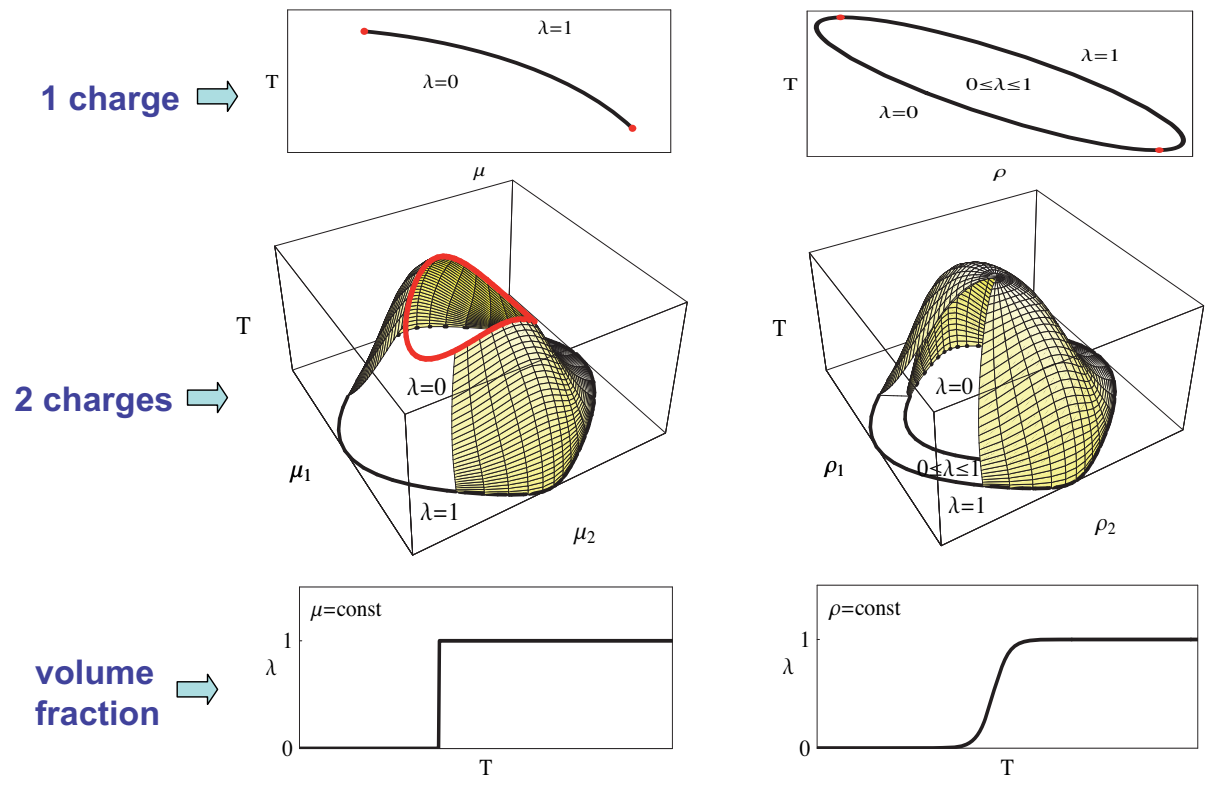

A.Sissakian, A.Sorin, V.Toneev, nucl-th/0608032 


\section{"Pasta" structures (finite size effects)}

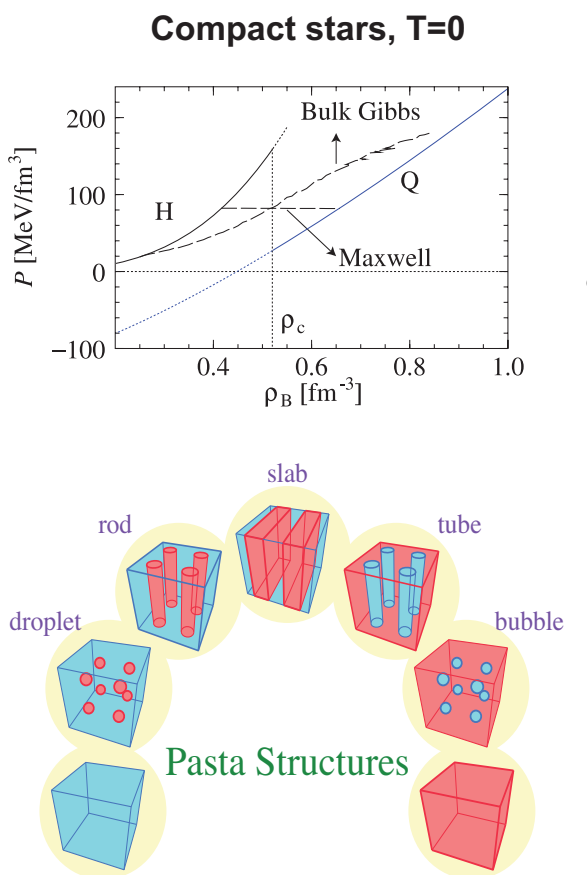

T.Maruyama et al., nucl-th/0605075

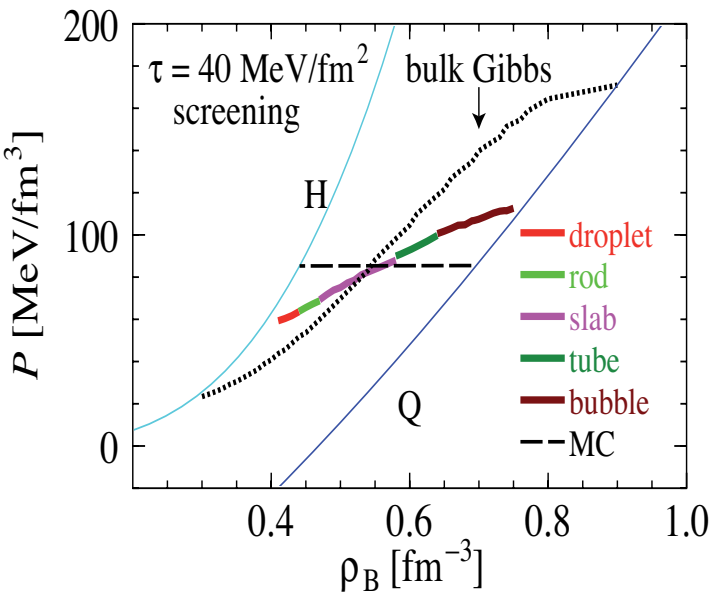

Charge density and particle density are non-uniform

\section{Dynamics of the first order phase transition}

Evolution of a gas babble in a mixed phase. Van der Waals equation of state.

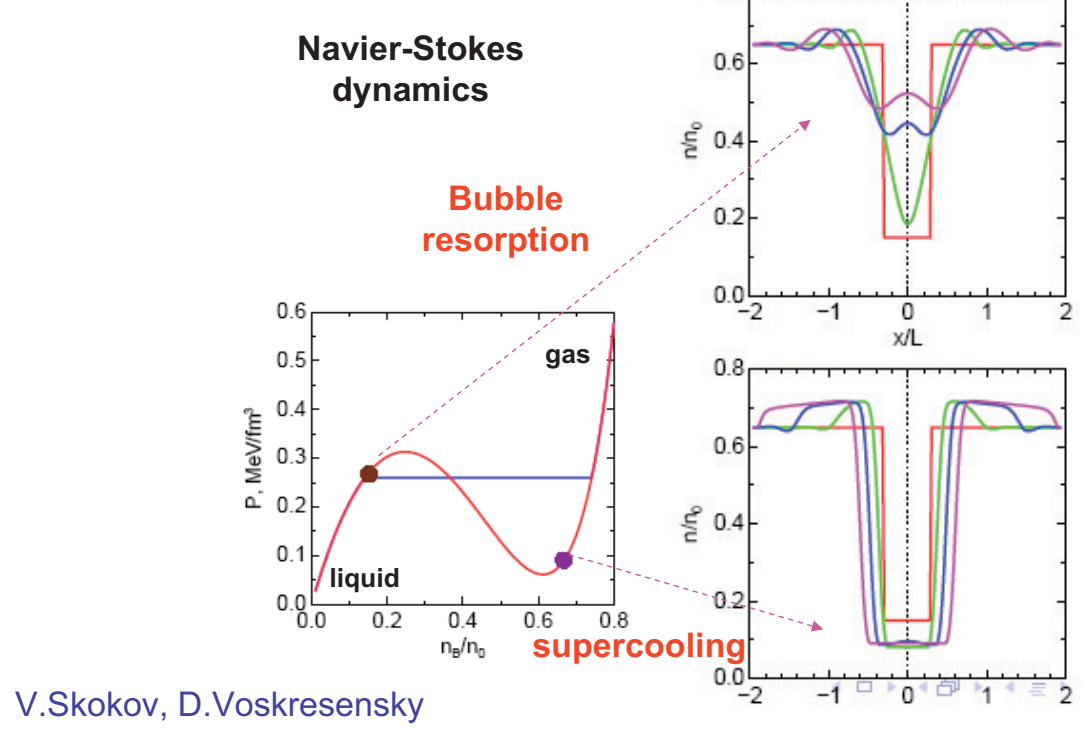




\section{Evolution of a mixed phase}
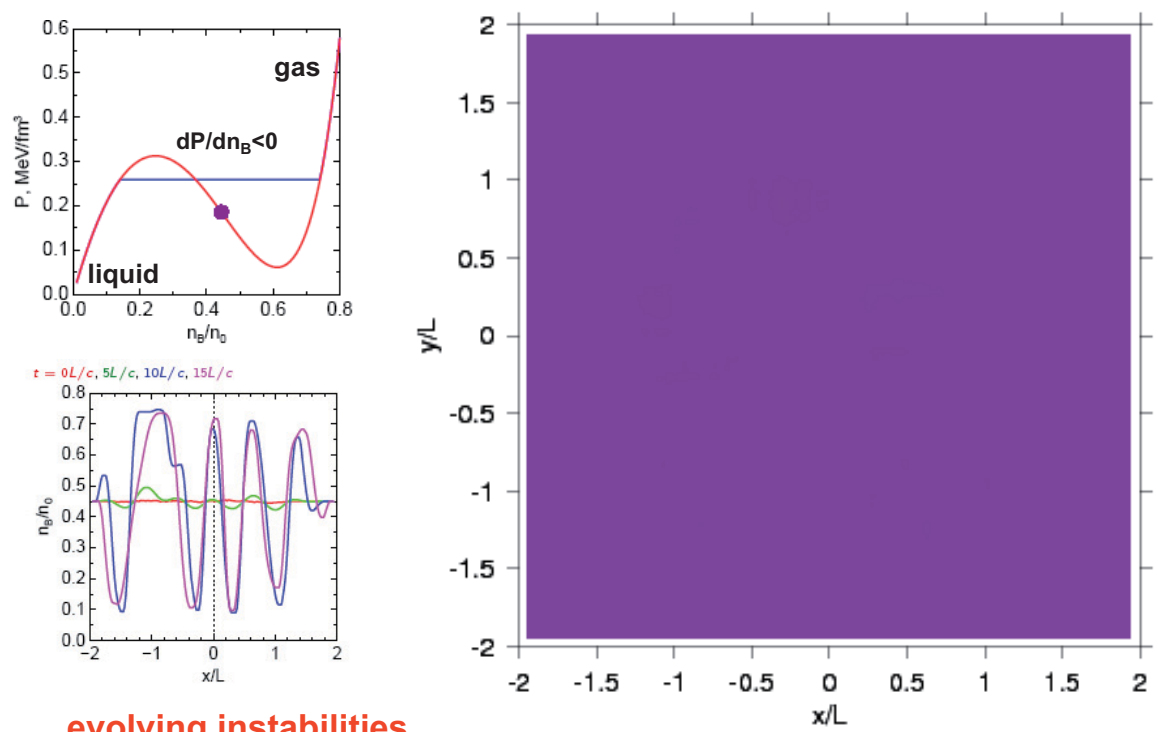

evolving instabilities

http://www-linux.gsi.de/ skokov/playme.gif

\section{Heat capacity (nuclear matter)}

$$
\begin{gathered}
T^{-1}=\frac{\partial S}{\partial E} \quad C_{i}^{-1}=-T^{2} \frac{\partial^{2} S}{\partial E^{2}} \\
E_{t}=E_{1}+E_{2} \quad T_{1}=T_{2}=T \\
C_{t} \approx C_{1}+C_{2}=\frac{C_{1}^{2}}{C_{1}-\sigma_{1}^{2} / T^{2}}
\end{gathered}
$$

Statistical Tsallis model of
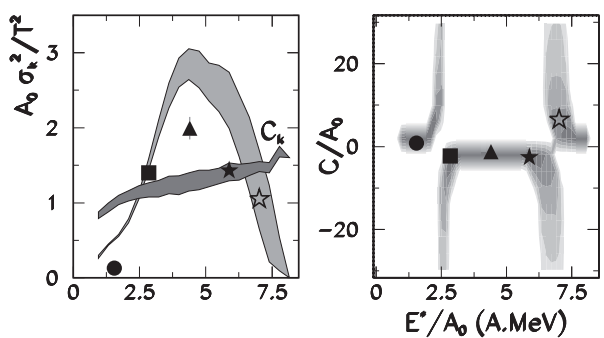

heat capacity is negative in the mixed phase

M.D'Agostino et al. NP A734 (2004) 512

\section{multifragmentation}

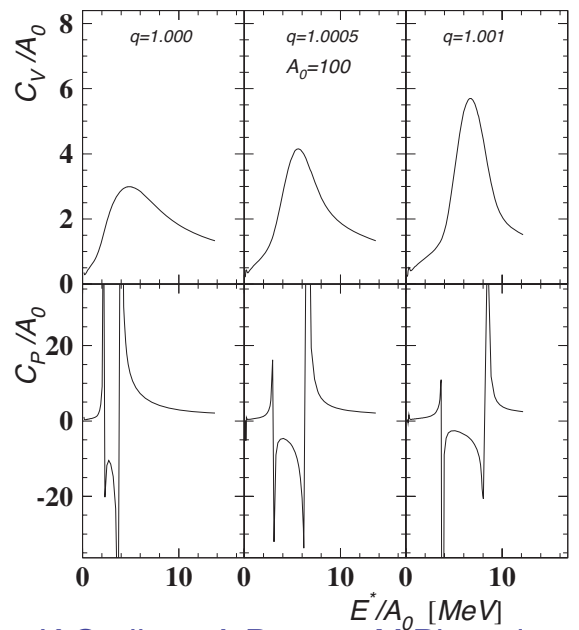

K.Gudima, A.Parvan, M.Ploszajczak

V.Toneev, PRL 85 (2000) 4691 


\section{Susceptibility}

Nambu--Jona-Lasinio model

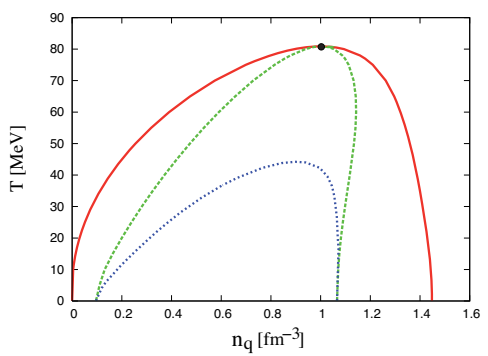

$$
\chi_{q}=-\frac{1}{V} \frac{\partial^{2} F}{\partial \mu_{q}^{2}}=\frac{1}{V}\left(<N^{2}>-<N>^{2}\right)
$$

$\mid \frac{\partial P}{\partial V}=0$ : isothermal $\quad \mathbf{T}_{\mathrm{c}}=81 \mathrm{MeV}$

$\left.\overline{\partial V}\right|_{T}=0$ : $\mathbf{\mu}=330 \mathrm{MeV}$

$\left.\frac{\partial P}{\partial V}\right|_{S}=0:$ isentropic

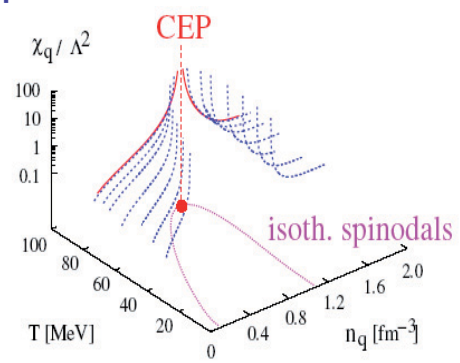

poles, negative branch

instability region shrinks toward the critical end point

K.Redlich, B.Friman, C.Sasaki,

J. Phys. G34 (2007) 437

\section{Distillation in the mixed phase}

for unbound quarks in a fireball

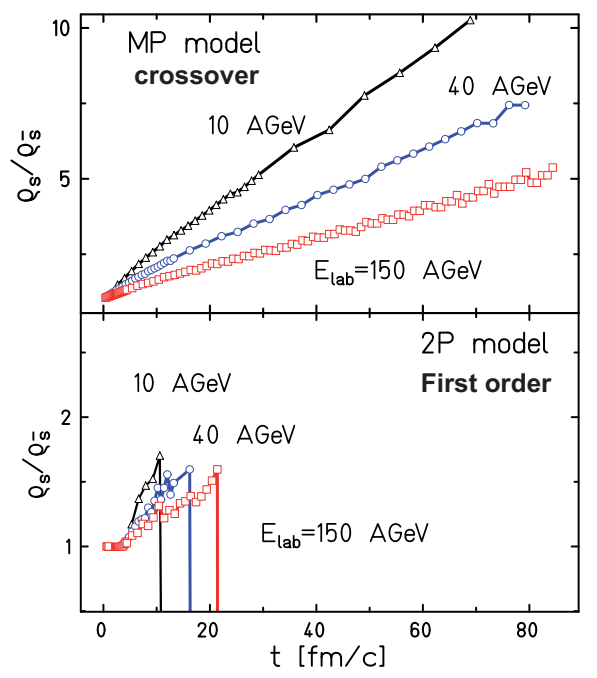

strangeness separation

V.Toneev et al., Eur. Phys. J. C32 (2004) 415.
$\mathrm{K}^{+} / \pi^{+}$excitation function, statistical equilibrium along the freeze-out line

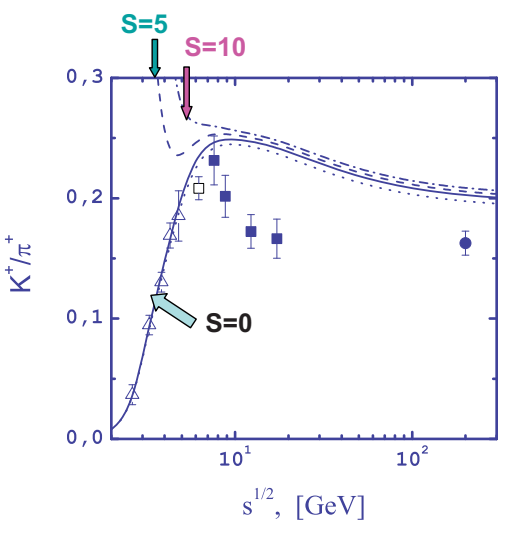

At $\mathrm{CP}$ the $\mathrm{K}^{+} / \pi^{+}$ratio should jump down on the $S=0$ curve

V.Toneev, A.Parvan, J. Phys. G: Nucl. Part. Phys. 31 (2005) 583 


\section{Lattice QCD calculations (history)}

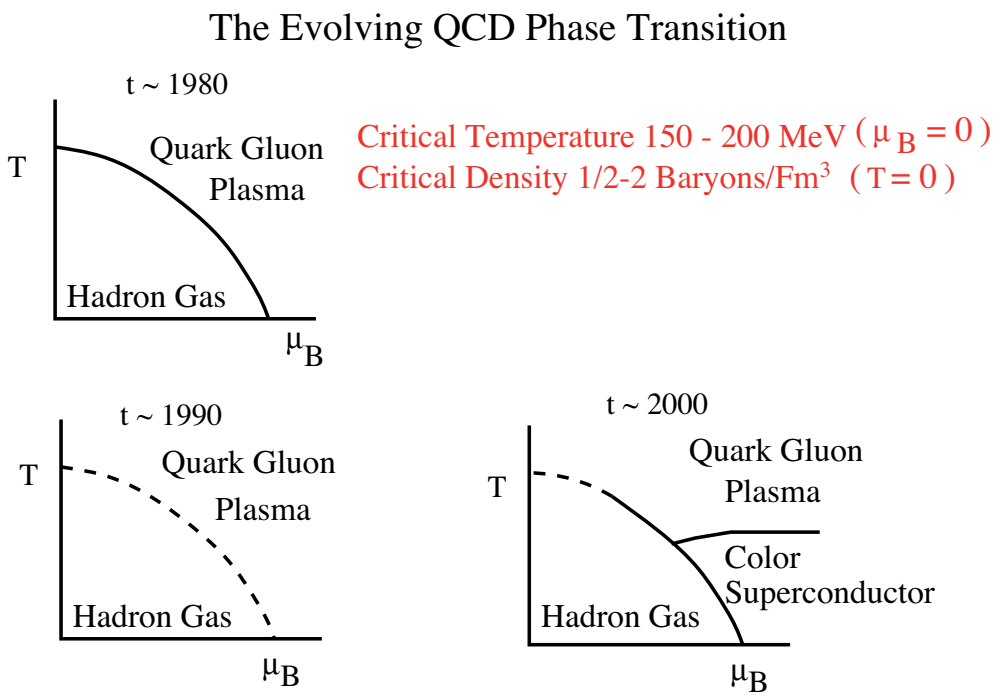

L. McLerran, Prama 60 (2003) 575

\section{Lattice QCD predictions for the order of PT}

E.Laermann, O.Phillipsen, Ann.Rev.Nucl.Part. Sci.,51 (2003) 163

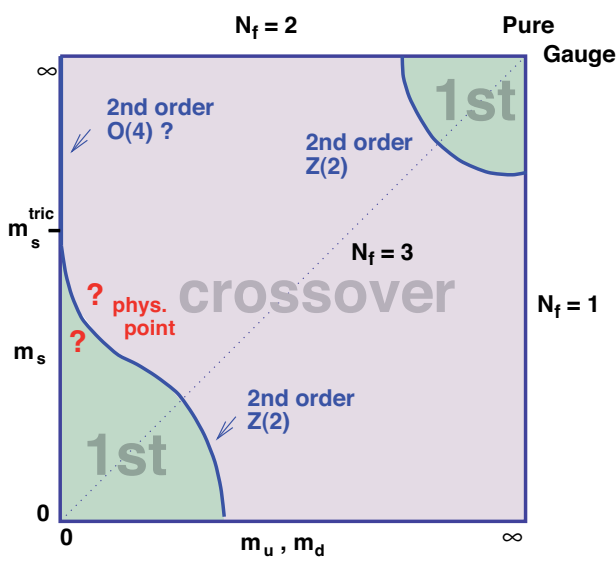

$$
\mu_{B}=0
$$

\section{S.Kim et al. hep-lat/510069}

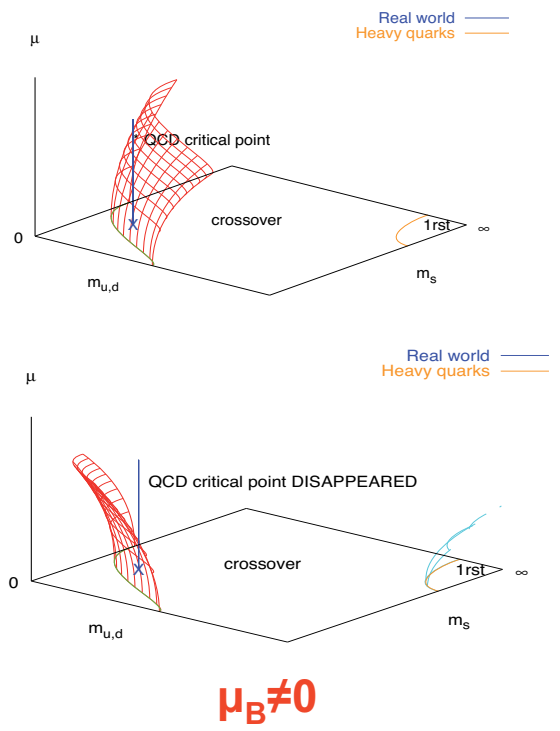




\section{Time evolution near the QCD critical point}

Two-phase EoS (first order)
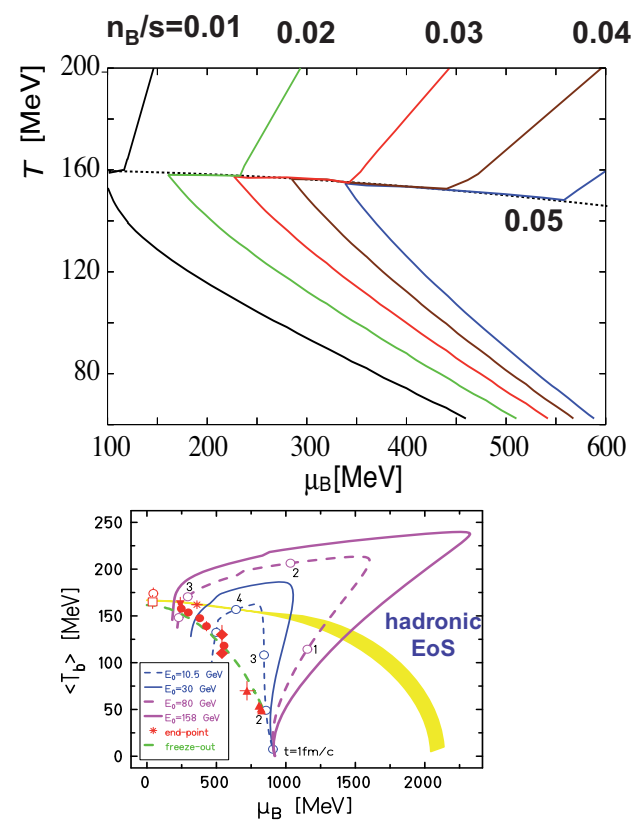

\section{A glance at the collision dynamics}




\section{Evolution of nucleus-nucleus collisions}
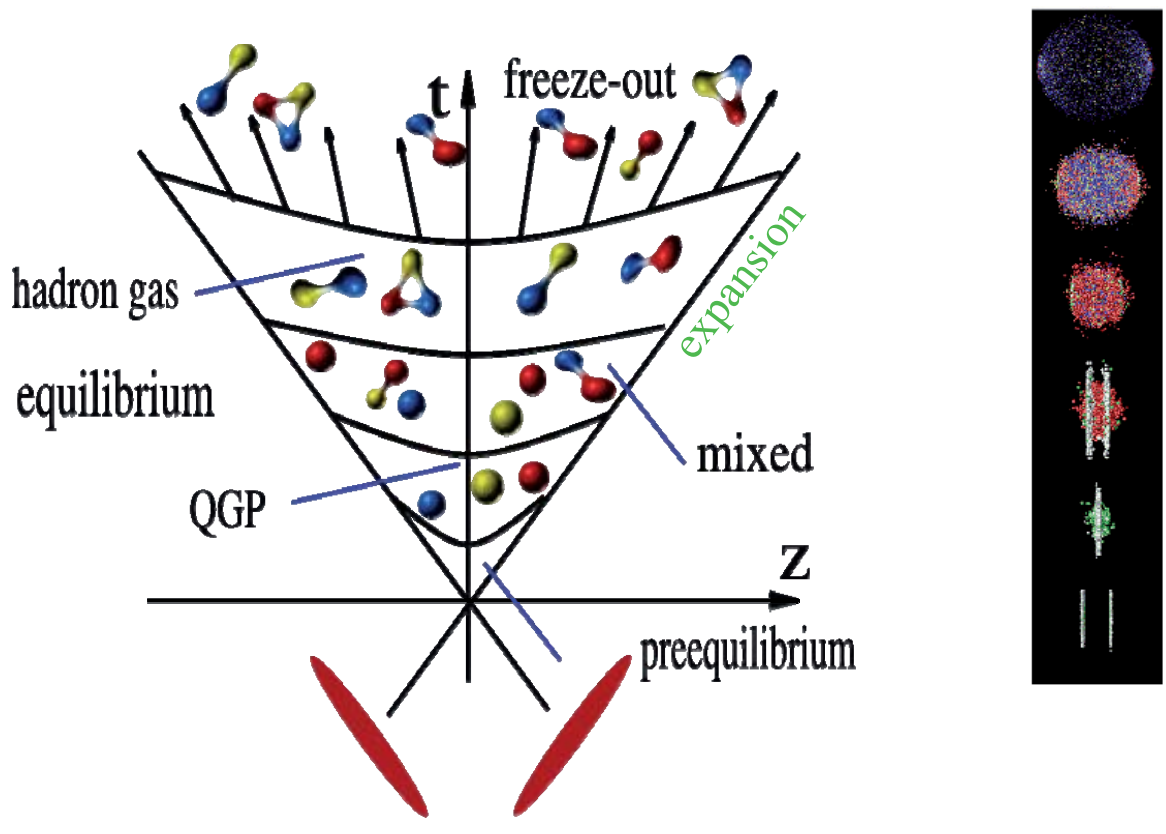

\section{Nuclear interaction scales}

$d$ - repulsion $N N$ force range

$\Lambda=\frac{1}{\sigma \rho}-$ (nucleon) mean free path $\rho_{0} \simeq 0.16 \mathrm{fm}^{-3} \sigma \simeq 40 \mathrm{mb} \rightarrow \Lambda \sim$ $1.5 \mathrm{fm}$

\section{Pauli principle} compression $L$ - "macroscopic" length, 2-8 fm

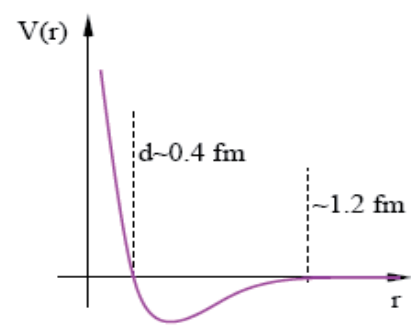

\begin{tabular}{|l|ccc|c|c|}
\hline units & $\mathrm{d}$ & $\wedge$ & $\mathrm{L}$ & $\mathrm{d} / \wedge$ & $K n=\wedge / L$ \\
\hline air $\left(10^{-8} \mathrm{~cm}\right)$ & 1 & $10^{5}$ & $10^{8}$ & $10^{-3}$ & $10^{-3}$ \\
liquid $\left(10^{-8} \mathrm{~cm}\right)$ & 1 & $2-10$ & $10^{8}$ & $0.1-0.5$ & $10^{-7}$ \\
nuclei $(0.4 / 1.2 \mathrm{fm})$ & 1 & $1.5-2$ & $2-8$ & $0.2-0.6$ & $1-0.2$ \\
\hline
\end{tabular}

$$
\text { kinetics } \Leftarrow \quad d \ll \wedge \ll L \quad \Rightarrow \text { hydrodynamics }
$$

For nuclear case (intermediate energies) : $\quad d<\wedge<L$ 


\section{Relativistic Boltzmann equation}

$$
\begin{gathered}
\left(p_{\mu} \partial^{\mu}\right) f_{i}\left(x, p_{i}\right)=\sum_{j} C^{r e l}\left(x, p_{i}\right)+\sum_{r} R_{r \rightarrow i} \\
=-f_{i}\left(x, p_{i}\right) \sum_{j} \int d \omega_{j} f_{j}\left(x, p_{j}\right) Q_{i j} \sigma^{i j}+\sum_{k j} \int d \omega_{k} d \omega_{j} \Phi\left(p_{j} p_{k} \mid x, p_{i}, \tau_{f}\right) \\
+\sum_{r} \int d \omega_{k^{\prime}} d \omega_{r} f_{r}\left(x, p_{r}\right) \Gamma^{r \rightarrow i+k^{\prime}} \delta\left(p_{r}-p_{i}-k^{\prime}\right)
\end{gathered}
$$

hadron production rate

$$
\Phi\left(p_{j} p_{k} \mid x, p_{i}, \tau_{f}\right)=\int d x^{\prime} \underbrace{f_{k}\left(x^{\prime}, p_{i}\right) f_{j}\left(x^{\prime}, p_{j}\right) Q_{i j} \sigma^{i j}}_{\text {collision rate }} \underbrace{\phi\left(x^{\prime} \mid x, p_{i}, \tau_{f}\right)}_{\text {transition prob. }}
$$

with $d \omega=d^{3} p / E, Q_{i j}=\left(p_{i} p_{j}\right)^{2}-p_{i}^{2} p_{j}^{2}=\left|v_{i}-v_{j}\right| E_{i} E_{j}$ transition probability for a finite formation time

$$
\begin{aligned}
& \phi\left(x^{\prime} \mid x, p, \tau_{f}\right)=\frac{1}{\sigma} \frac{d \sigma}{d \omega} \theta\left(t-t^{\prime}-\tau_{f}\right) \delta^{(3)}\left(\vec{x}-\vec{x}^{\prime}-\frac{\vec{p}}{E}\left(t-t^{\prime}\right)\right) F\left(\tau_{f}\right) \\
& \text { * multiple particle production } \Rightarrow \text { coupled set of equations for } \\
& \text { stable hadrons and resonances }\left\{h_{i}\right\} \text {; new flavors } \\
& \star \text { finite formation time } \theta\left(t-\tau_{f}\right), \tau_{f}=(E / m) \tau_{f}^{0} \text { with } \tau_{f}^{0} \sim 1 \mathrm{fm} \text {; }
\end{aligned}
$$

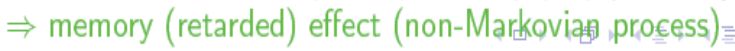

- new degrees of freedom (QCD) : quark/gluons, strings, formation of color rope

\section{* Hadron as a string}

\section{G.S.Bali, K.Schilling, Phys.Rev.D 46 (1992) 2636}

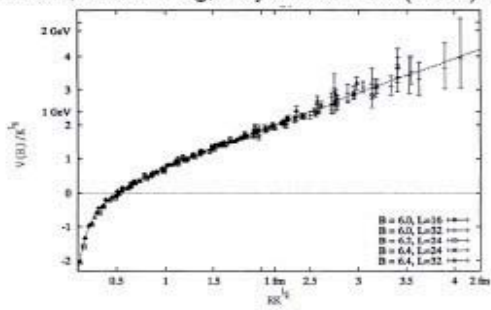

$$
\begin{aligned}
& V_{q \bar{q}}=-\frac{\alpha_{e f f}}{r}+\kappa r \\
& H_{y o-y o}=\left|p_{1}\right|+\left|p_{2}\right|+\kappa\left|x_{1}-x_{2}\right| \\
& \frac{d p_{1,2}}{d t}= \pm \kappa, \frac{d x_{1,2}}{d t}= \pm 1 \\
& x^{+}=\frac{p^{+}}{\kappa}=\frac{E+p}{\kappa}, x^{-}=\frac{p^{-}}{\kappa}=\frac{E-p}{\kappa}, S=\frac{p^{+} p^{-}}{\kappa^{2}}=\frac{E^{2}-p^{2}}{\kappa^{2}}=\frac{m^{2}}{\kappa^{2}}
\end{aligned}
$$

yo-yo mode

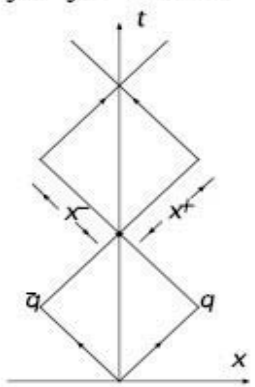


- Particularities of space-time evolution

\section{CLASSICAL STRING THEORY}

$\star$ string fusion

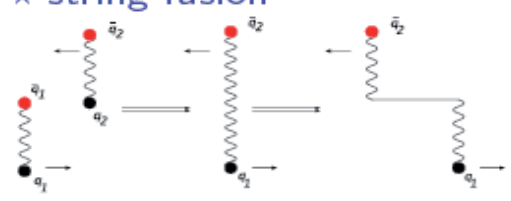

$$
\text { time } \Rightarrow
$$

$\star$ string rearrangement

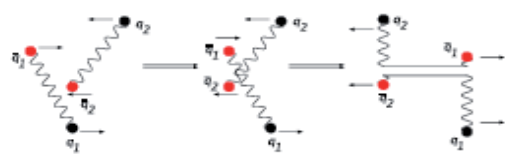

* leading particle effect

$\star$ color rope formation
DUAL TOPOL. MODEL

* planar diagram

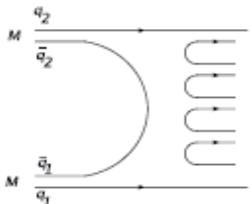

$\star$ cylindrical diagram

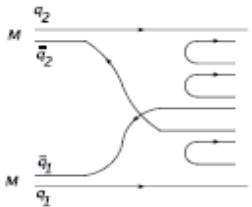

\section{Kinetic results}

QGSM model

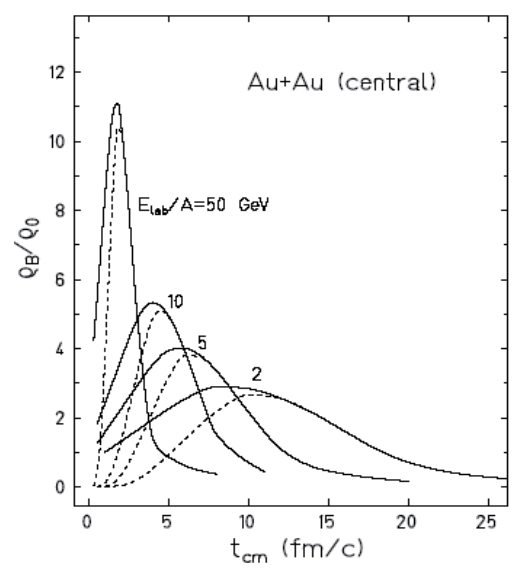

I.C.Arsene et al., Phys. Rev. C75 (2007) 034902

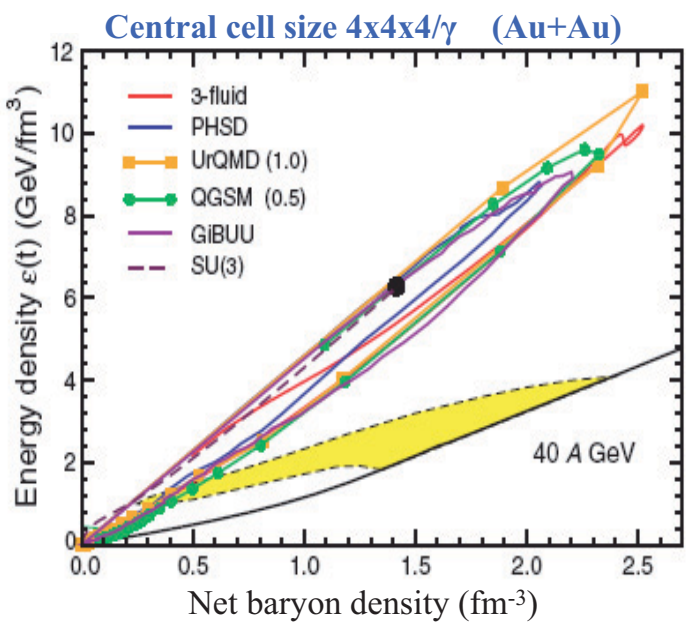

All dynamical models (w/o PT) demonstrate similar results 


\section{Rapidity distributions}
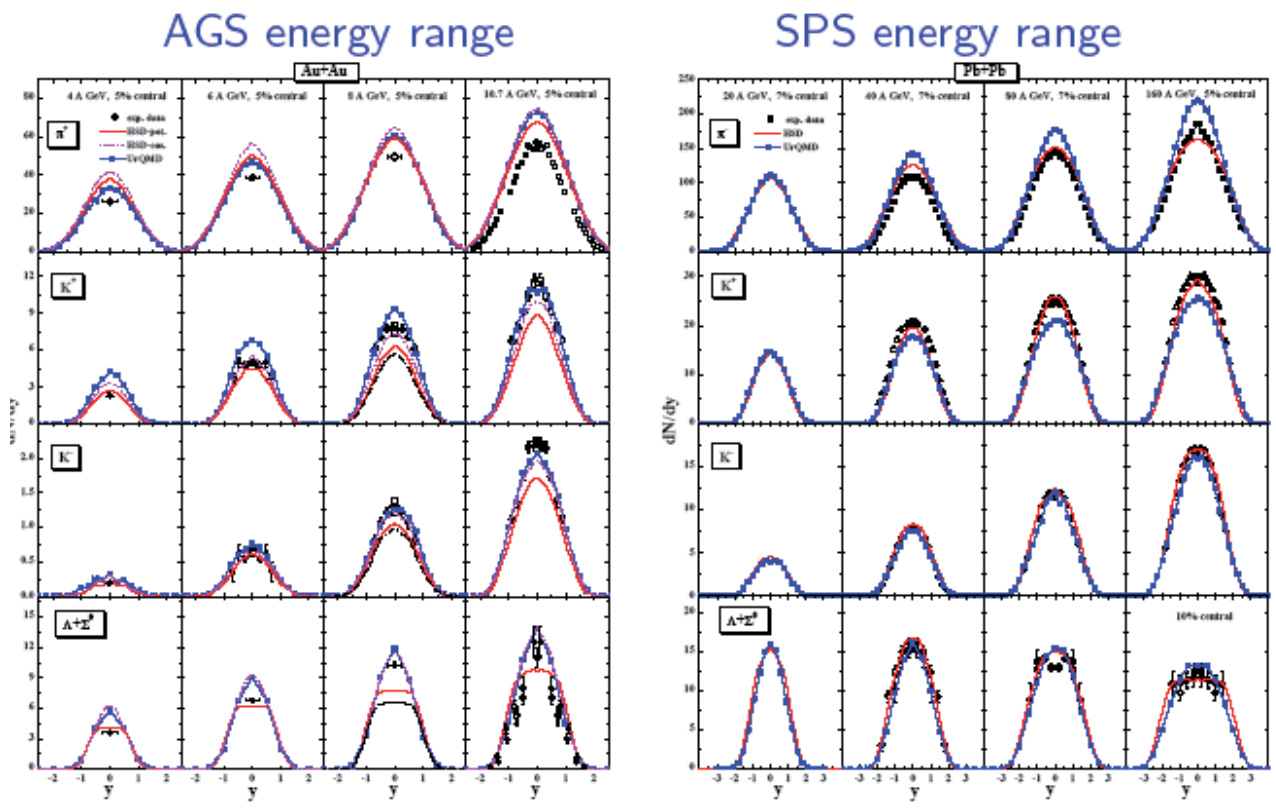

H.Weber et al., Phys.Rev. C67 (2003) 014904

\section{Hydrodynamic approach}

- Conservation laws (Gauss theorem) $\Rightarrow$ Fluid dynamics

$$
\begin{array}{rlr}
\partial_{\mu} J_{i}^{\mu} & =0 \text { net charge } i \text { conservation } \\
\partial_{\mu} T^{\mu \nu} & =0 \text { energy momentum conservation } & 4
\end{array}
$$

- Tensor decomposition of the charge current $J^{\mu}$ and energy-momentum tensor $T^{\mu \nu}$ with respect to 4-velocity $u^{\mu}$

$$
\begin{aligned}
& \begin{aligned}
& J_{i}^{\mu}=n_{i} u^{\mu}+\left(g_{\nu}^{\mu}-u^{\mu} u_{\nu}\right) J_{i}^{\nu}+\ldots \\
& T^{\mu \nu}=\varepsilon u^{\mu} u^{\nu}-P\left(g^{\mu \nu}-u^{\mu} u^{\nu}\right)+q^{\mu} u^{\nu}+q^{\nu} u^{\mu}+\pi^{\mu \nu}+\ldots \\
& \text { Navier-Stockes }
\end{aligned} \\
& \qquad \begin{aligned}
\text { with } J_{i}^{\mu} & =\int \frac{d^{3} p}{p_{0}} p^{\mu}\left[f_{i}(x, p)-\bar{f}_{i}(x, p)\right] \\
T^{\mu \nu} & =\int \frac{d^{3} p}{p_{0}} p^{\mu} p^{\nu}[f(x, p)+\bar{f}(x, p)] \\
f_{i}(x, p) & =\frac{g_{i}}{(2 \pi)^{3}} \exp \left[\left(\left(u_{\mu} p^{\mu}(x)-\mu_{i}(x)\right) / T(x)\right) \pm 1\right]^{-1}
\end{aligned}
\end{aligned}
$$




\section{Gradient expansion}

$\star$ Perfect hydro in local thermodynamical equilibrium

$$
\begin{aligned}
J_{i}^{\mu} & =n_{i} u^{\mu} \\
T^{\mu \nu} & =\underbrace{\varepsilon u^{\mu} u^{\nu}-P\left(g^{\mu \nu}-u^{\mu} u^{\nu}\right)}_{\text {perfect hydro }} \\
& + \text { EoS } \varepsilon(p)
\end{aligned}
$$

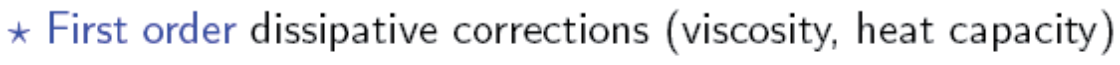

$$
\Rightarrow \text { acausality }
$$

Navier-Stokes

* Second order corrections $\Rightarrow+14$ Grad equations

Israel-Stuart

Spatial-temporal variation of the macro fields has to be SMALL

\section{Relation between kinetics and hydrodynamics}

- The non-relativistic case (for nucleons)

$\int d^{3} p\left[\begin{array}{c}\rho \\ \vec{v} \\ \epsilon\end{array}\right] \frac{d}{d t} f(\vec{p}, \vec{x}, t)=\int d^{3} p\left[\begin{array}{c}1 \\ \vec{p} / m_{N} \\ p^{2} / 2 m_{N}\end{array}\right]$ । $(\vec{p}, \vec{x}, t)$

Boltzmann equation + local equilibrium hypothesis

$$
\begin{array}{rlrl}
\vec{v} & =\vec{u}+\vec{c}, \text { hydro } \vec{u}=<\vec{v}>, \text { thermo }<\vec{c}>=0 \\
\rho<c_{i} c_{k}> & =P \delta_{i k}+\Pi_{i k}, \quad \rho<c^{2} c_{k}>=Q_{k} & \\
\frac{\partial \rho}{\partial t}-\frac{\partial}{\partial x_{k}} \rho u_{k}=0 & & \\
\frac{\partial \rho u_{i}}{\partial t}-\frac{\partial}{\partial x_{k}} \rho u_{i} u_{k} & =\left\{\frac{\partial}{\partial x_{k}} \Pi_{i k}-\frac{\partial}{\partial x_{i}} P\right\} & & \rho, \epsilon, P \\
\frac{\partial \epsilon}{\partial t}-\frac{\partial}{\partial x_{k}} \epsilon u_{k}=\left\{\frac{\partial}{\partial x_{k}} \Pi_{i k} u_{i}-\frac{\partial}{\partial x_{i}} P u_{k}-\frac{\partial}{\partial x_{k}} Q_{k}\right\} & & \text { EOS }
\end{array}
$$

hydro: $\Lambda \ll L ; R e=\frac{\text { inertial }}{\text { viscous }} \simeq \frac{M}{\Lambda / L} \simeq 4-10$ with $M=v / c_{s}$ and $c_{s}=\left.\sqrt{\partial P / \partial \rho}\right|_{s} \approx 0.2 \quad$ turbulent regime $R e \simeq 10^{2}-10^{3}$ 


\section{Main ingredients of hydro approach}

\section{Non-equilibrium and initial state}

- to postulate an initial state (Landau, Bjorken, ...)

- to calculate it in kinetic models (QGSM, UrQMD)

- to introduce many-fluid dynamics $f(x, p)=\sum_{j}^{M} f_{j}^{e q}(x, p)$

A single fluid may consist of several particle spaces.

Different fluids may be of the same particle spaces.

$$
\begin{array}{cc}
\text { Equation of state } \quad(\text { hadronic EoS }) & \text { Yu.B.Ivanov, } \\
\varepsilon\left(n_{B}, T\right)=\varepsilon_{\text {gas }}\left(n_{B}, T\right)+W\left(n_{B}\right), & \text { V.N.Russkikh, } \\
P\left(n_{B}, T\right)=P_{\text {gas }}\left(n_{B}, T\right)+n_{B} \frac{d W\left(n_{B}\right)}{d n_{B}}-W\left(n_{B}\right) & \text { V.D.Toneev } \\
\text { Phys.Rev C73 } \\
\text { (2006) } 044904
\end{array}
$$

3-fluid hydro:

nuclear potential $W\left(n_{B}\right)=n_{B} m_{N}\left[-b\left(\frac{n_{B}}{n_{0}}\right)+c\left(\frac{n_{B}}{n_{0}}\right)^{\gamma+1}\right]$

Freeze-out procedure ( locally $\left.\varepsilon>\varepsilon_{\mathrm{f}}\right)$

\section{3-fluid hydrodynamic model}

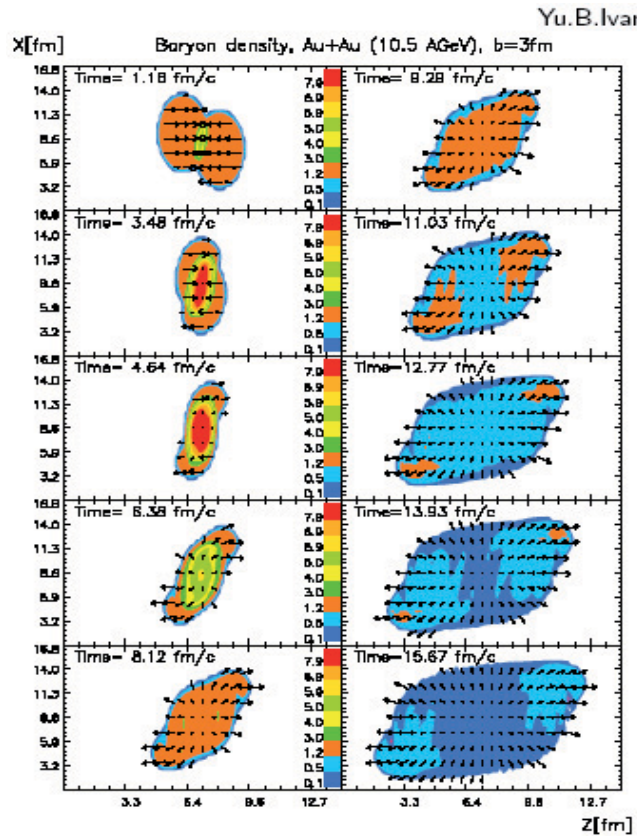

$x$ [fm] Boryonrich fluid energy density. Au+fus (10.5 AGeV), $b=3 \mathrm{fm}$

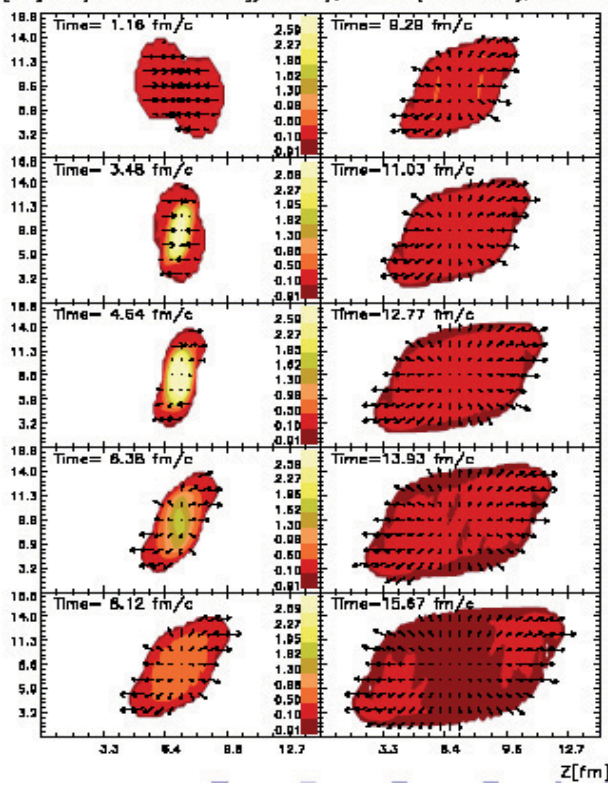




\section{3-fluid hydrodynamic model, cont'd}
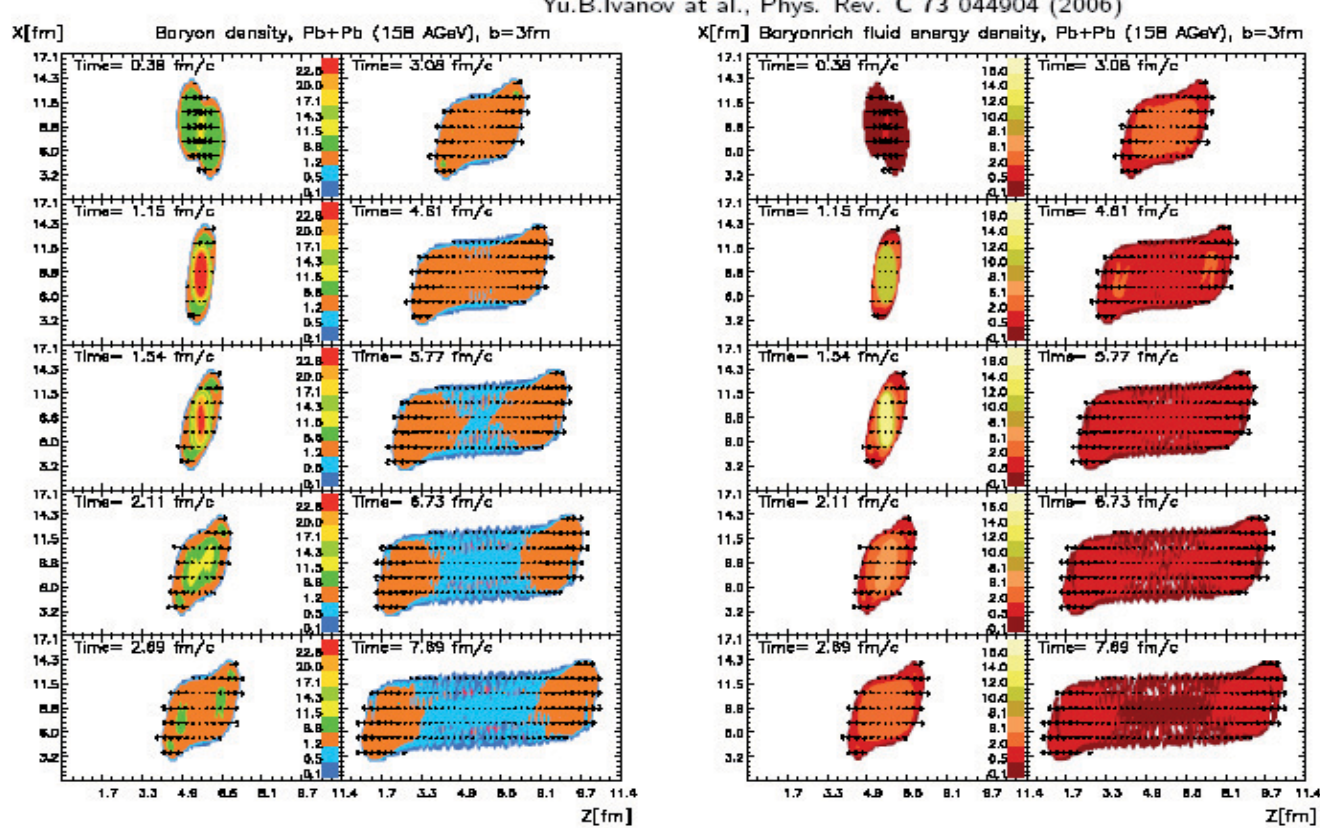

\section{Phase diagram (hadronic EoS)}

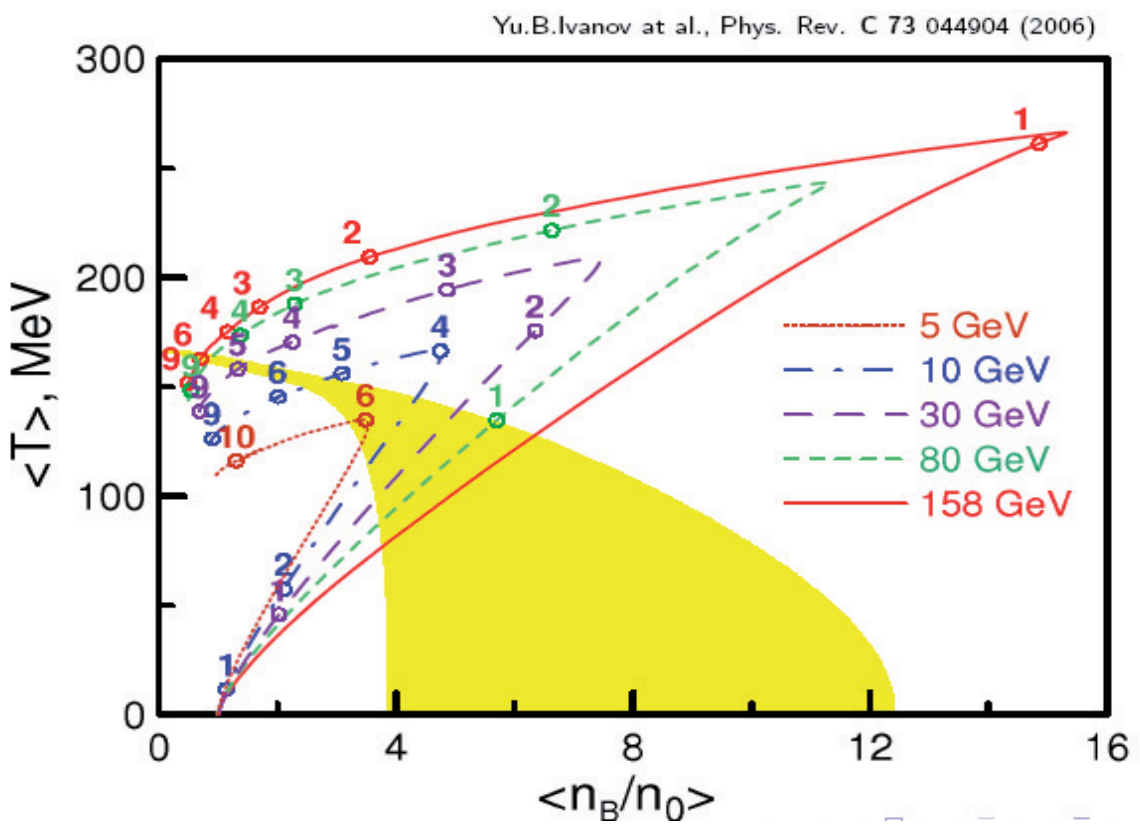




\section{Mean particle multiplicity}

* Freeze out ( local $\left.\varepsilon<\varepsilon_{f}\right) \Rightarrow$ observable
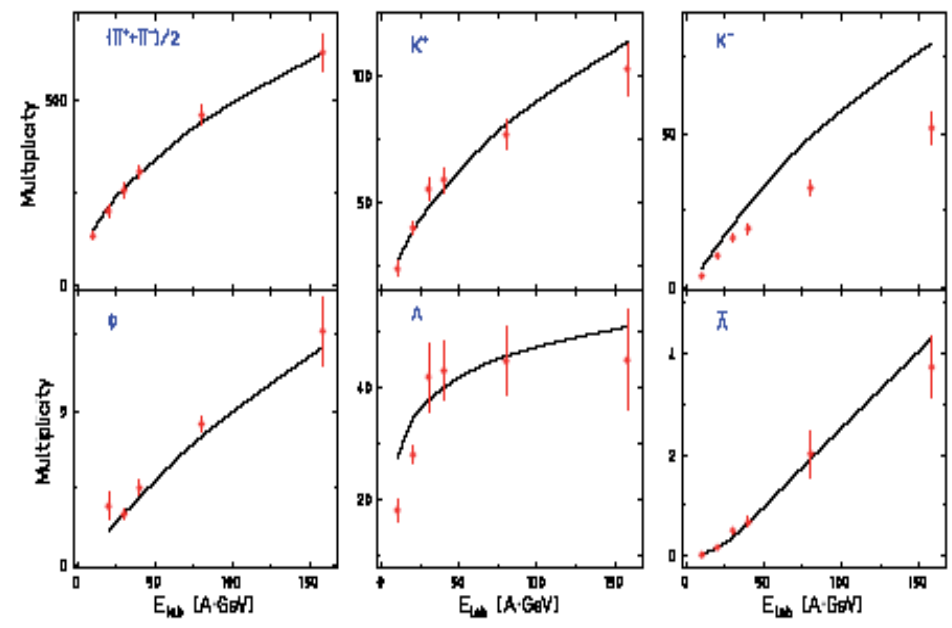

\section{Hybrid model:}

* initial state of nucleusnucleus interaction

Entropy evolution and a fireball formation are calculated within the transport code QGSM which defines an initial state for subsequent hydrodynamic stage for participant (central collisions)

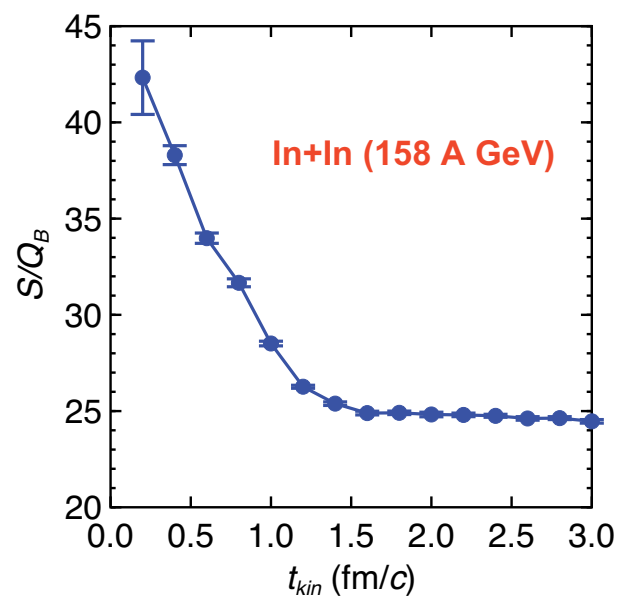




\section{Hybrid model, cont'd}

* Hydrodynamic expansion stage Isentropic expansion of the formed fireball is described by the 3D relativistic hydrodynamics with the above calculated energy and baryon densities as well as the velocity profile. The statistical quark-hadron equation of state with crossover phase

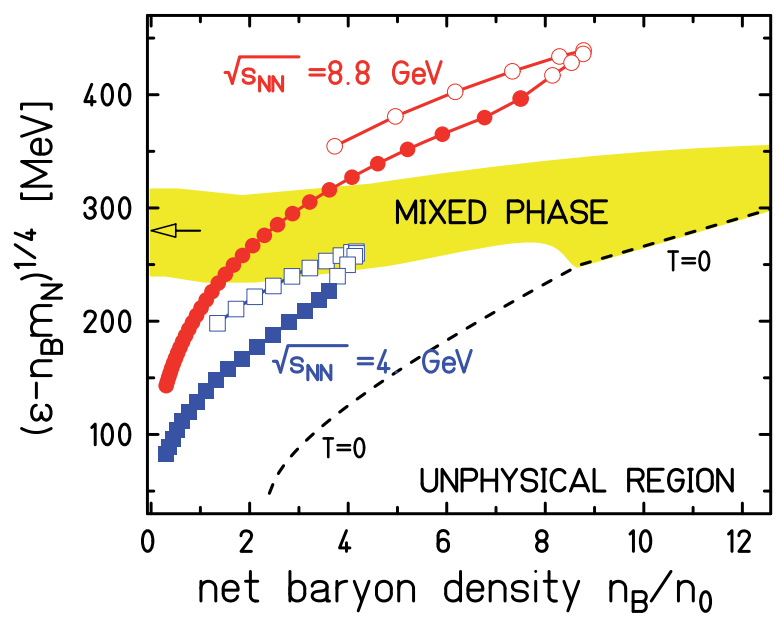

transition is used.

\section{Status of signals of a phase transition in HIC}




\section{Onset of the deconfinement} entropy $S \sim n_{\pi}$
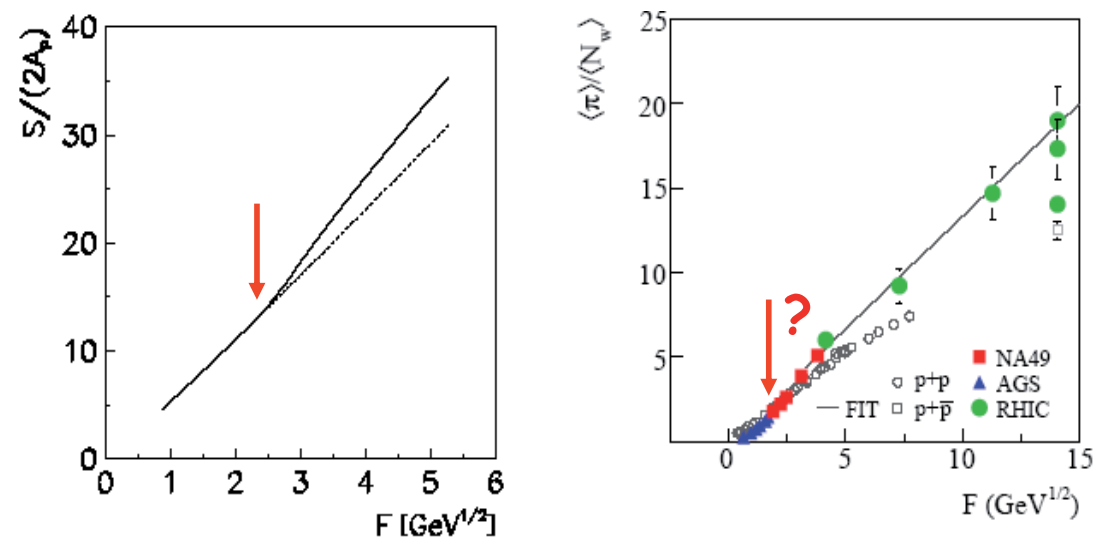

$F=\left(\sqrt{s_{N N}-2 m_{N}}\right)^{3 / 4} /\left(s_{N N}\right)^{1 / 4} \approx 2.25 \mathrm{GeV}^{1 / 2}$

$\left(E_{\text {beam }} \approx 30 \mathrm{AGeV}\right)$

M.Gazdzicki, arXiv:0712.3001

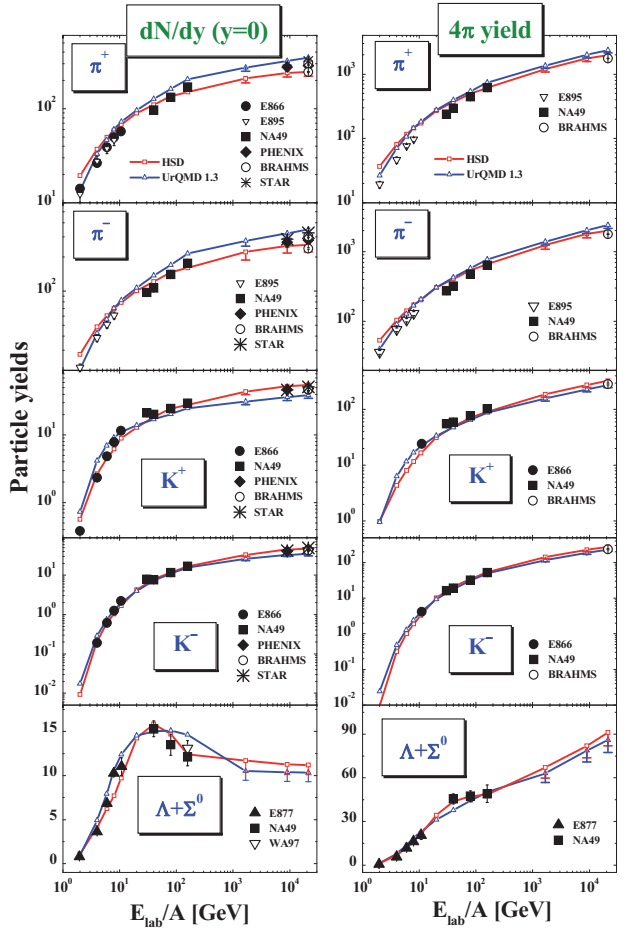

Excitation functions of $\pi^{ \pm}, K^{ \pm},\left(\Lambda+\Sigma^{0}\right)$ yields

Pion yield is overestimated by HSD below AGS energy and by UrQMD at the top AGS and above (deviations

$$
\text { are }<20 \% \text { ). }
$$

Reasonable description of strangeness by both models.

E.Bratkovskaya 


\section{Strangeness enhancement}

- Strangeness production in a hadronic world (at low energy):

$\mathrm{N}+\mathrm{N} \rightarrow \mathrm{N}+\Lambda+\mathrm{K}$ requires $\Delta \mathrm{E}=2 \mathrm{M}_{\mathrm{N}}-\left(\mathrm{M}_{\mathrm{K}}+\mathrm{M}_{\Lambda}+\mathrm{M}_{\mathrm{N}}\right)=670 \mathrm{MeV}$

$\pi+\mathrm{N} \rightarrow \Lambda+\mathrm{K} \quad \Delta \mathrm{E}=\left(\mathrm{M}_{\pi}+\mathbf{M}_{\mathrm{N}}\right)-\left(\mathrm{M}_{\mathrm{K}}+\mathrm{M}_{\Lambda}\right)=535 \mathrm{MeV}$

- Strangeness production in a QGP:

bare mass of strange quark $m_{S} \sim 150 \mathrm{MeV}$

$=>$ s-sbar pair production

by $q$-qbar annihilation $q+q b a r->s+s b a r$ needs only $\Delta E=300 \mathrm{MeV}$

=>s-sbar pair can be also produced by gluon fusion $\mathbf{g}+\mathrm{g} \rightarrow \mathrm{s}+\mathbf{s b a r}$

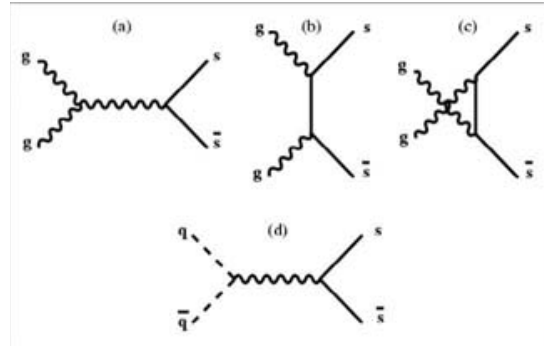

Strong enhancement of strangeness production in a QGP !

Rafelski-Müller: Phys. Rev. Lett. 48 (1982) 1066

$=>$ strangeness enhancement increases with strangeness content stronger effect for multi-strange hadrons $\Xi$ (uss), $\Omega($ sss)

\section{Strange-to-nonstrange particle ratio}
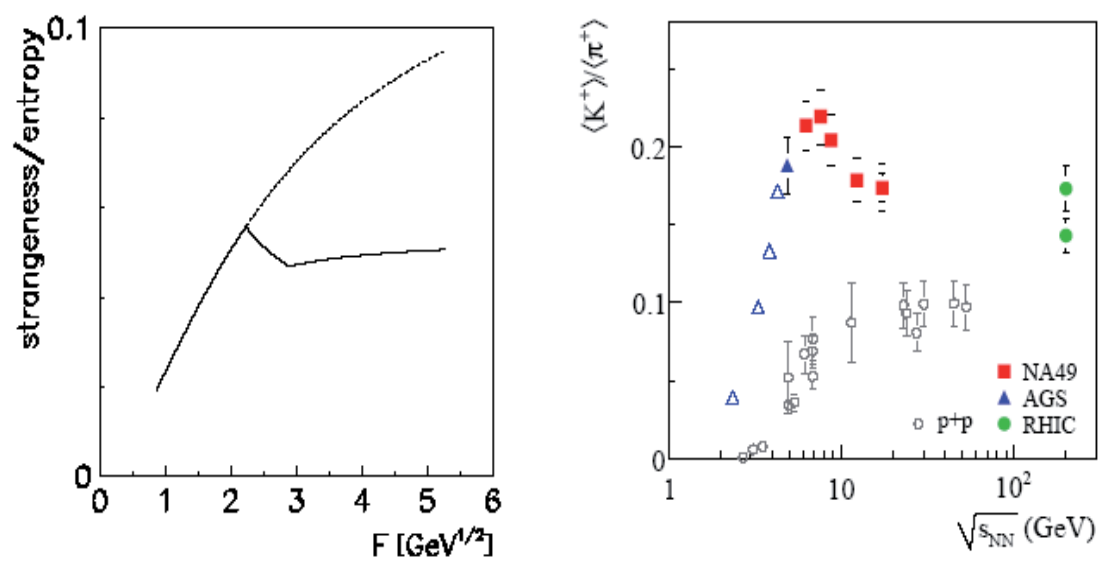

M.Gazdzicki, arXiv:0712.3001 
Excitation functions of $\mathrm{K}^{+} / \pi^{+}, \mathrm{K}^{-} / \pi^{-}$and $\left(\Lambda+\Sigma^{0}\right) / \pi$ ratios
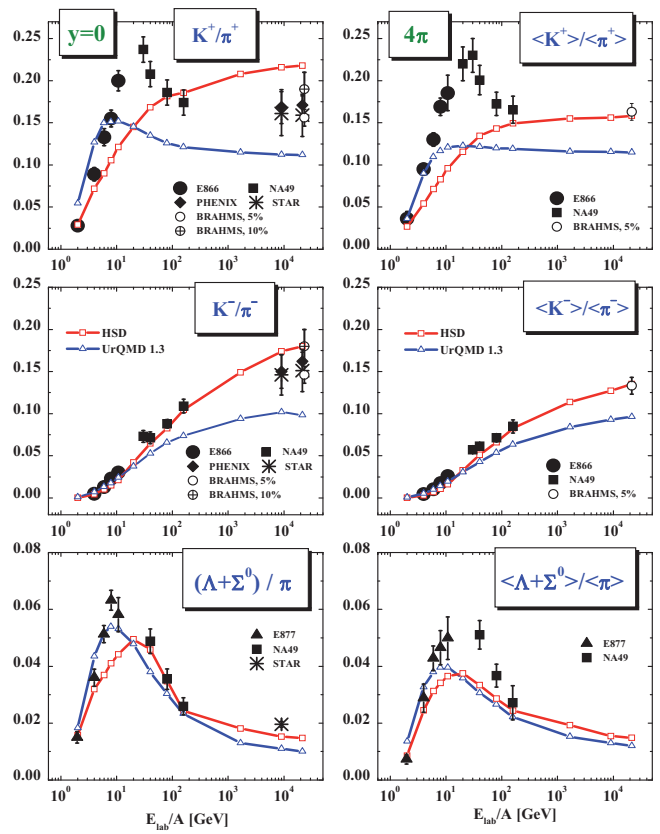

Experimental $\mathrm{K}^{+} / \pi^{+}$ratios show a peak at $\sim 30 \mathrm{~A} \mathrm{GeV}$

("horn") which is not reproduced by hadronic transport models HSD and UrQMD

Hyperon enhancements at $160 A \mathrm{GeV} / c$
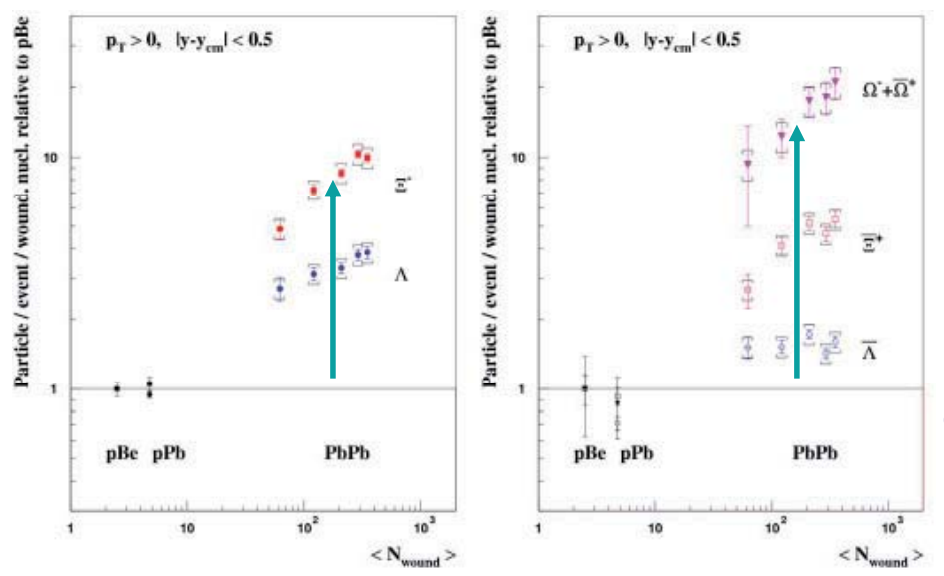

$\ulcorner$ systematic errors: $10 \%$ for $\Lambda, \Xi$ $15 \%$ for $\Omega$

Most peripheral class: $<N_{\text {wound }}>=62 \pm 4$

Significant centrality dependence of strangeness enhancements for all hyperons except for $\bar{\Lambda}$ 


\section{In-medium effect in RMF}

Baryon density and temperature dependence of hadron effective
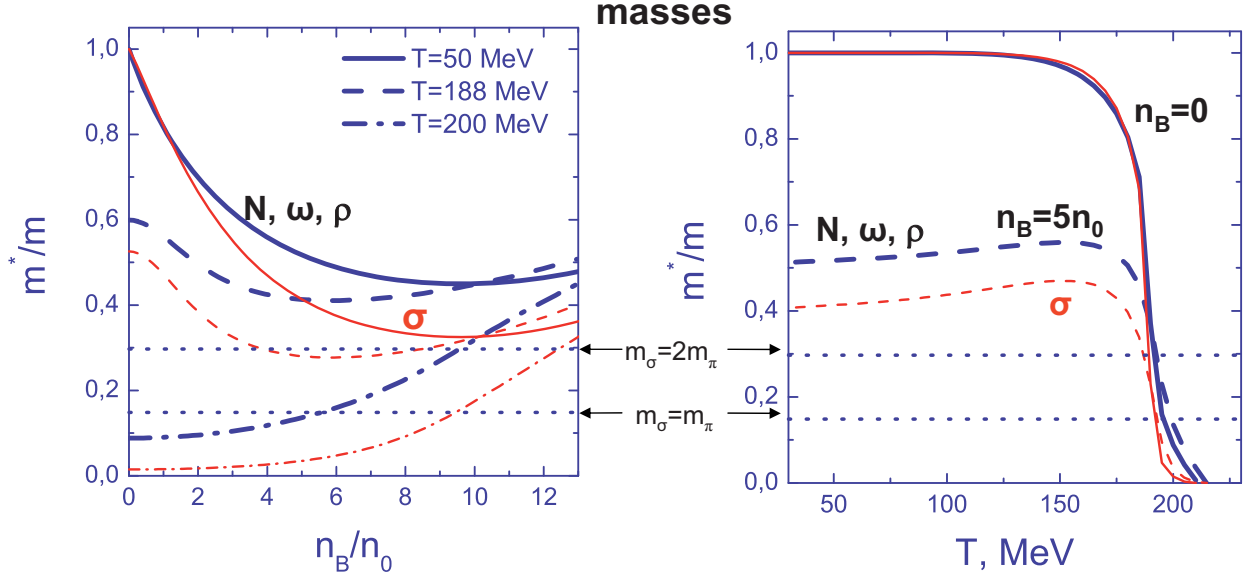

Relativistic mean-field model with scaled hadron masses and couplings simulates a partial restoration of the chiral symmetry

A.Khvorostukhin, V.Toneev, D.Voskresensky, Nucl. Phys. A791 (2007) 180

\section{In-medium (anti)particle number densities vs. $\mathrm{T}_{,} \mathrm{n}_{B}$}
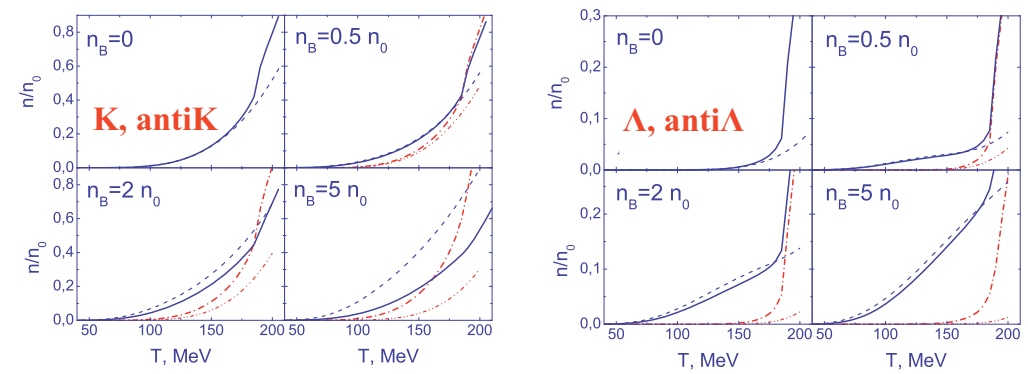

SHMC:

Solid =>

particles

Dashed dotted $=>$ antiparticles
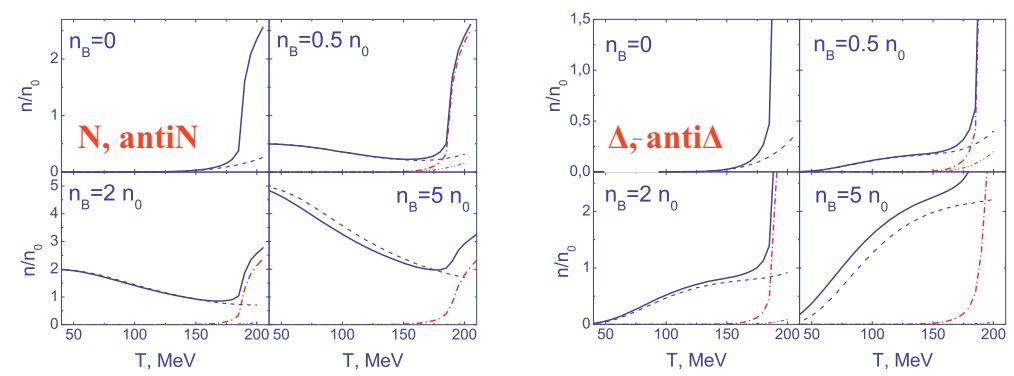

Ideal Gas:

Dashed $=>$ particles

Dashed double dotted $=>$ antiparticles

Strong increase of the hadron yield at T above $\sim 160 \mathrm{MeV}$ 


\section{Metastable Exotic Multistrange Object (W.Greiner)}

\section{MEMO $2 \mathrm{p} 2 \mathrm{n} 2 \wedge 2 \Sigma^{0} 2 \Xi^{-}$}

each baryon sits in the $1 \mathrm{~s}_{1 / 2}-\mathrm{s}$

PAULI BLOCKED!

$\rightarrow$ METASTABLE!

Properties:

Strangeness content $S / A=1$

Charge $z=\sigma$

Density $\rho \approx 4 \rho$

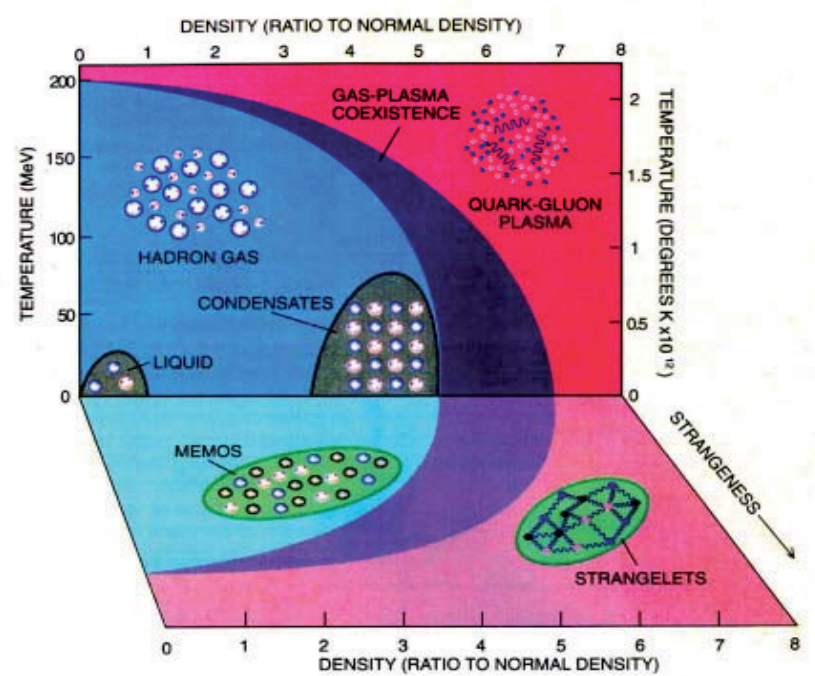

\section{Transverse temperature}
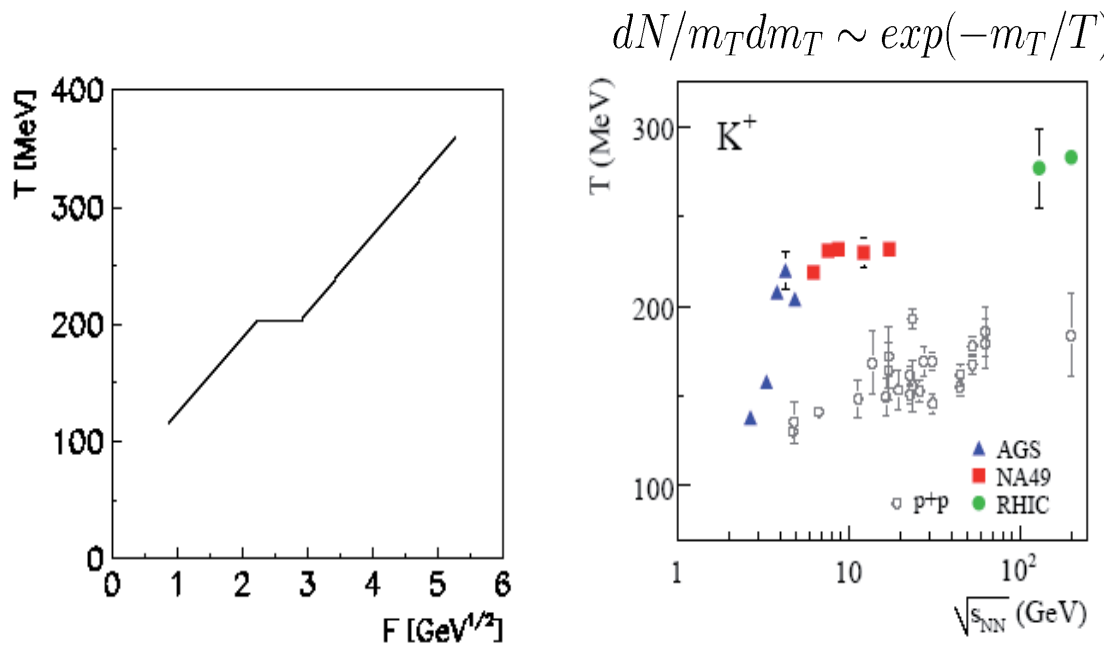

M.Gazdzicki, arXiv:0712.3001 

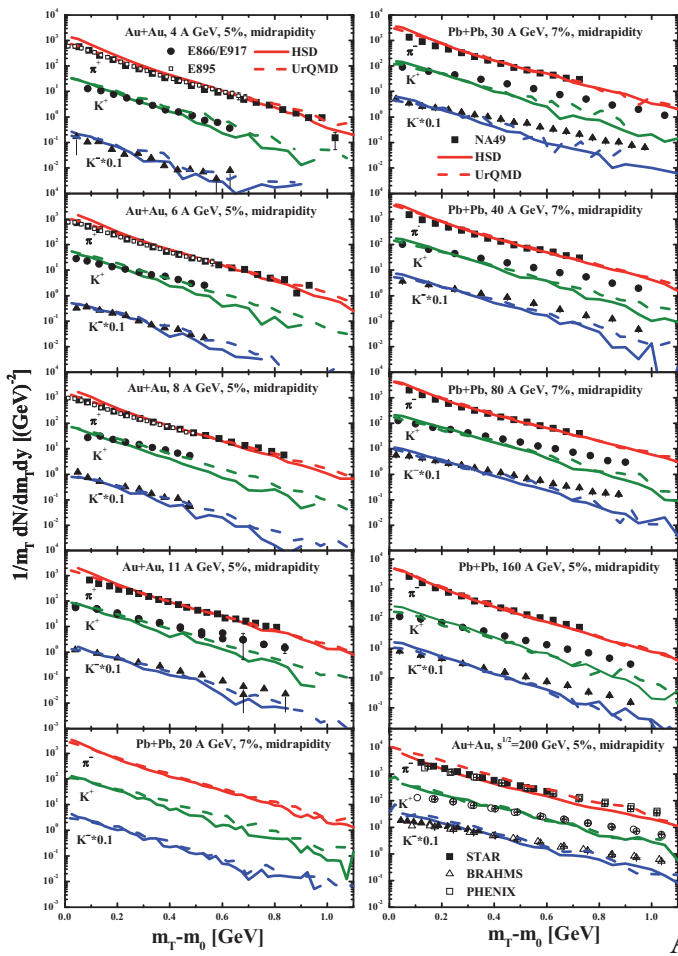

$m_{\mathrm{T}}$ spectra for central Au+Au collisions from AGS to RHIC energies

\author{
Pions slopes are only \\ slightly underestimated by \\ hadronic transport models \\ HSD and UrQMD
}

Kaon slopes are too small above 5 A GeV!

A.Mishra et al.,Phys. Rev. C69 (2004) 015202

\title{
Inverse $\mathrm{T}$ slopes of $\mathrm{K}^{+}$and $\mathrm{K}^{-}$spectra
}
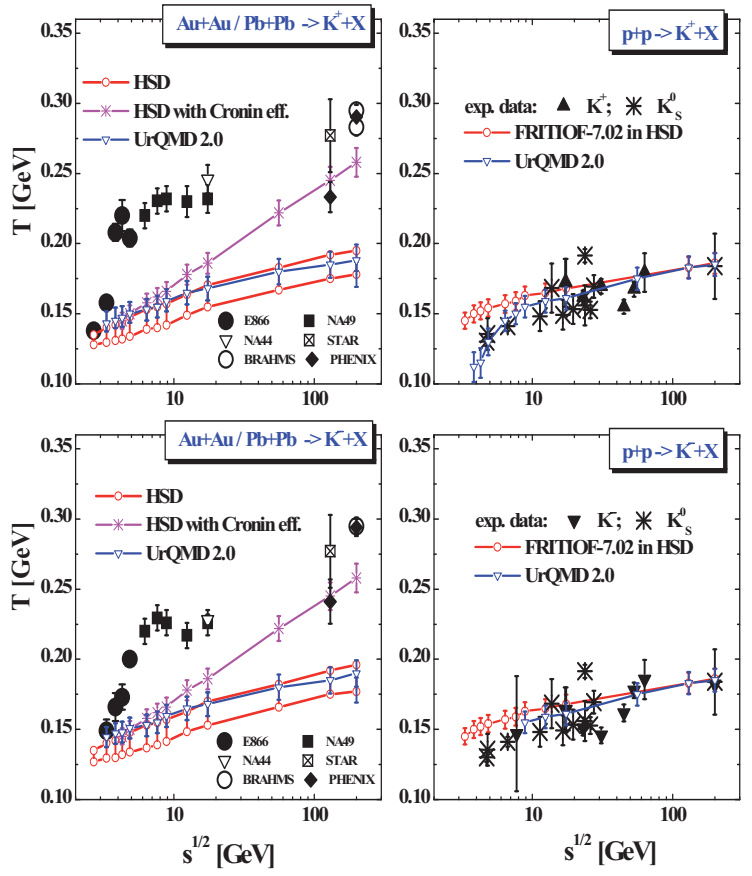

Phys.Rev. C69 (2004) 015202 PRL 92 (2004) 013302

In HSD and UrQMD hadronic rescattering has only a small impact on the slope of kaon spectra.

Cronin effect - initial semihard gluon radiation - leads to the substantial broadening of the $\mathrm{m}_{\mathrm{T}}$ spectra at RHIC, however, has a very small effect at low energies.

\section{The hadron-string} picture fails $? \rightarrow$ New degrees of freedom 


\section{3-fluid hydrodynamic model, central Au+Au}

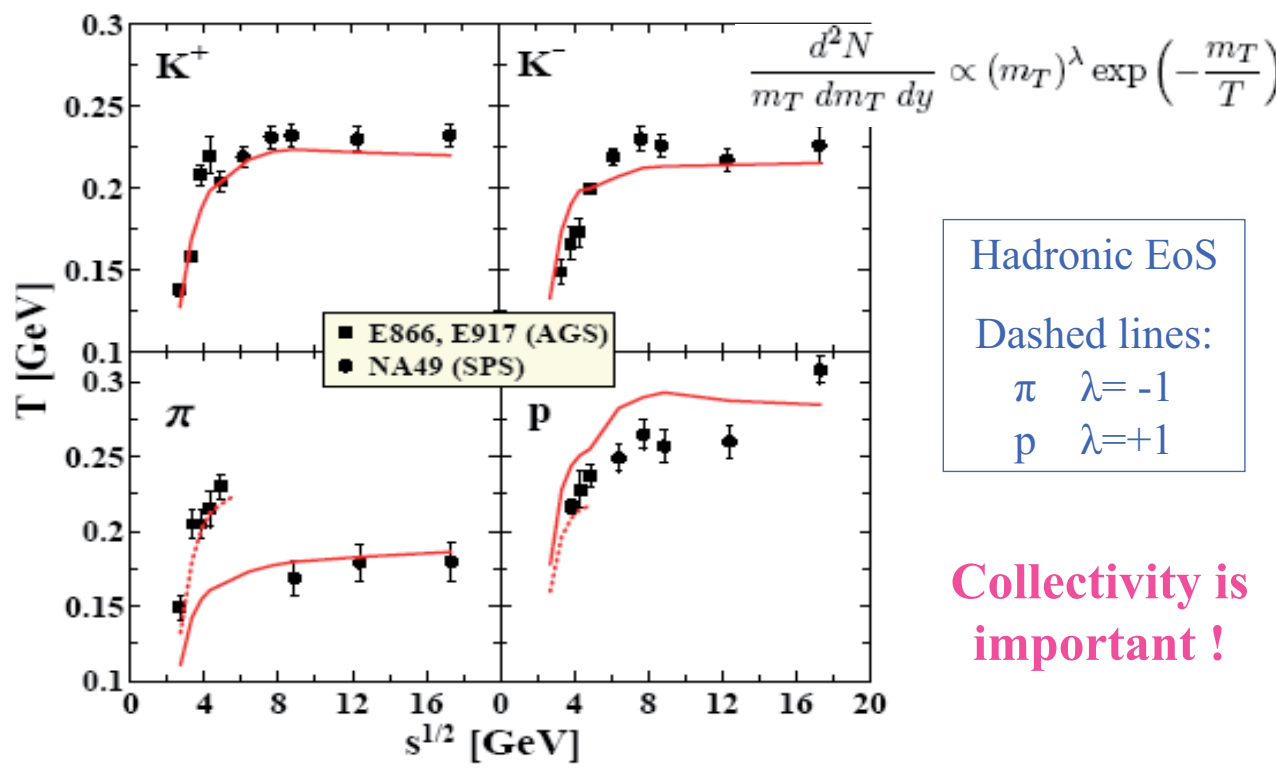

Yu.Ivanov, V.Russkikh, nucl-th/0607070

\section{Transport model with 3-body collisions}

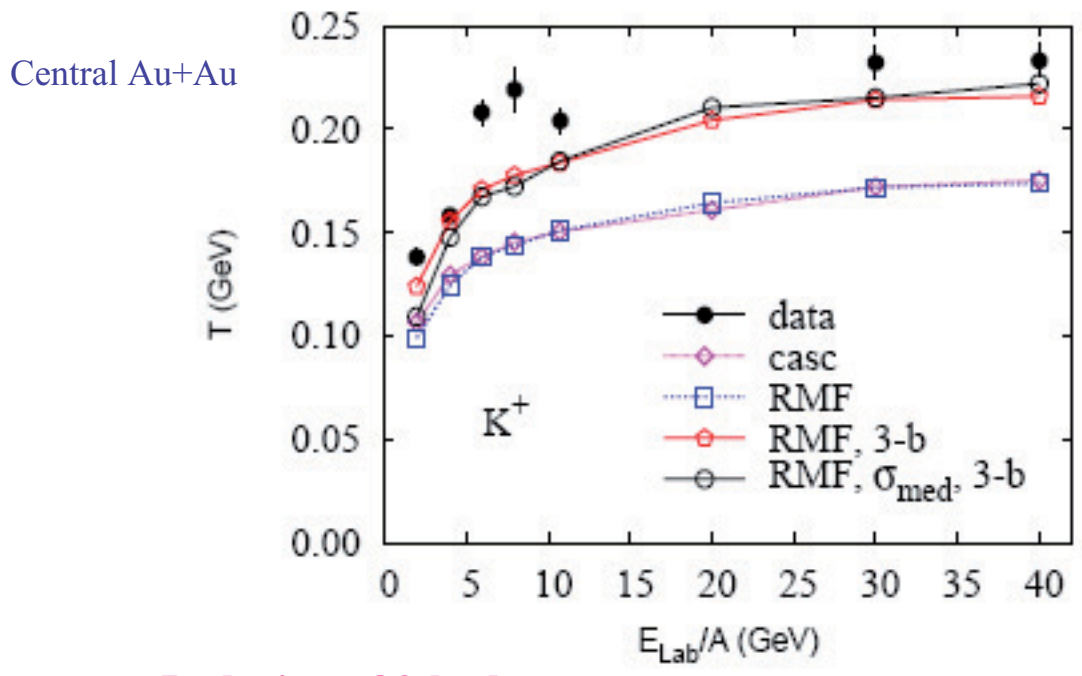

Inclusion of 3-body

collisions is important A.Larionov et al., Phys.Rev. C76 (2007) 044909 


\section{Remark: Blast-wave description of spectra}

\section{Model : thermalization plus hydro-dynamical transverse flow description}

$$
\begin{array}{cc}
\frac{\mathrm{d}^{2} N_{j}}{m_{T} \mathrm{~d} y \mathrm{~d} m_{T}}=\int_{0}^{R_{G}} A_{j} m_{T} \cdot K_{1}\left(\frac{m_{t} \cosh \rho}{T}\right) \cdot I_{0}\left(\frac{p_{t} \sinh \rho}{T}\right) r d r & \\
\rho(r)=\tanh ^{-1} \beta_{\perp}(r) & \text { Uniform particle density } \\
\beta_{\perp}(r)=\beta_{S}\left[\frac{r}{R_{G}}\right]^{n} \quad r \leq R_{G} & <\beta_{\perp}>=\frac{2}{2+n} \beta_{S}
\end{array}
$$

E Schnedermann, J Sollfrank and U Heinz, Phys. Rev. C48 (1993) 2462

Parameters: $\left\langle\beta_{\mathrm{T}}>, \mathrm{T}\right.$

\section{Blast wave description of the inverse slope $T$ values at 40 and $160 \mathrm{~A} \mathrm{GeV}$}
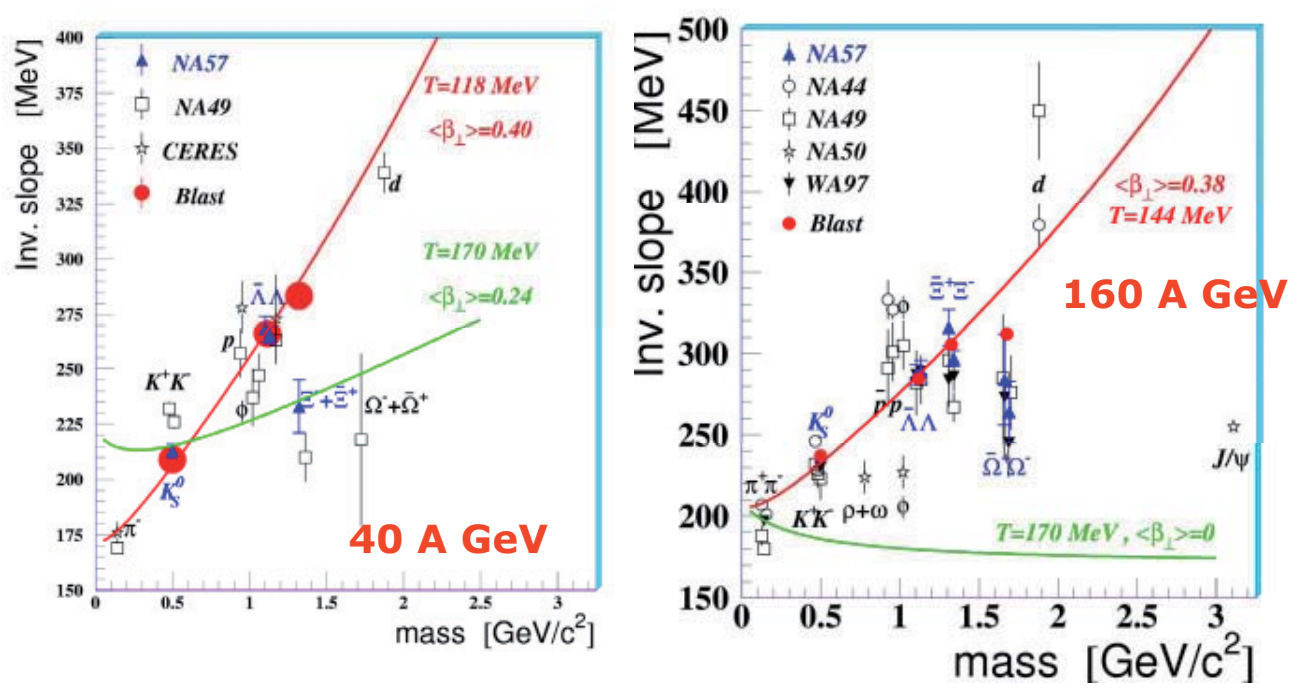

- indication of an early freeze-out of the $\Xi, \Omega$ 


\section{Fluctuations}

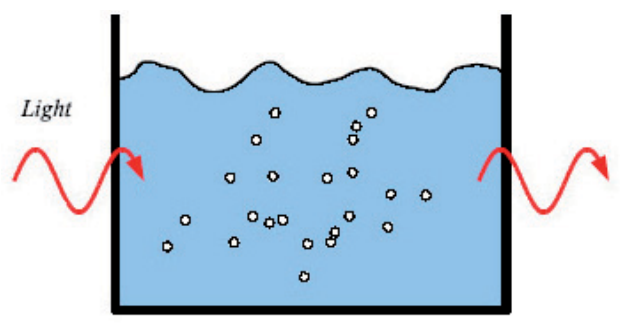

below the critical point

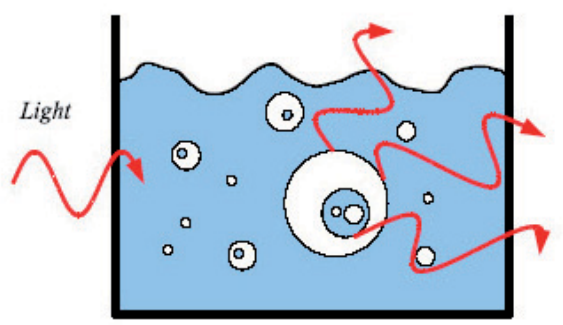

at the critical point $\triangleright$ in the vicinity of the critical point: all length scales are equally important.

$\triangleright$ fluctuation scales become arbitrarily large $\rightarrow$ measurable.

$\triangleright$ at the critical point:

light is strongly scattered.

\section{Fluctuations}

Lattice QCD predictions: Fluctuations of the quark number density (susceptibility) at $\mu_{B}>0$ (C.Allton et al., PR D68 (2003) 014507)

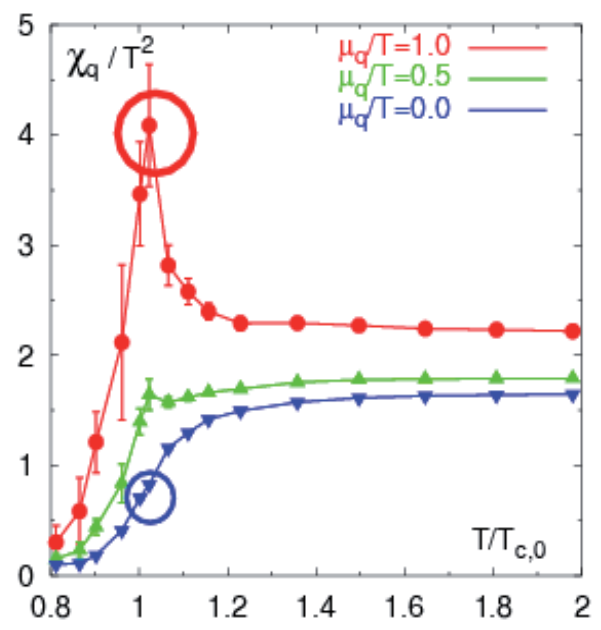

$$
\frac{\chi_{q}}{T^{2}}=\left[\frac{\partial^{2}}{\partial\left(\mu_{q} / T\right)^{2}} \frac{P}{T^{4}}\right]_{T_{f i x e d}}
$$

$X_{q}$ (quark number density

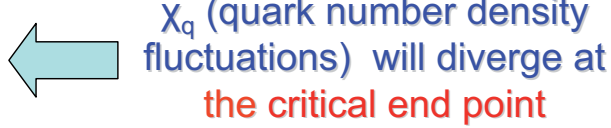

Experimental observation:

- Baryon number fluctuations

- Charge number fluctuations 


\section{Multiplicity fluctuations: experimental status}

$$
\omega_{i}=\frac{\left.<N_{i}\right\rangle^{2}-<N_{i}^{2}>}{<N_{i}>}
$$

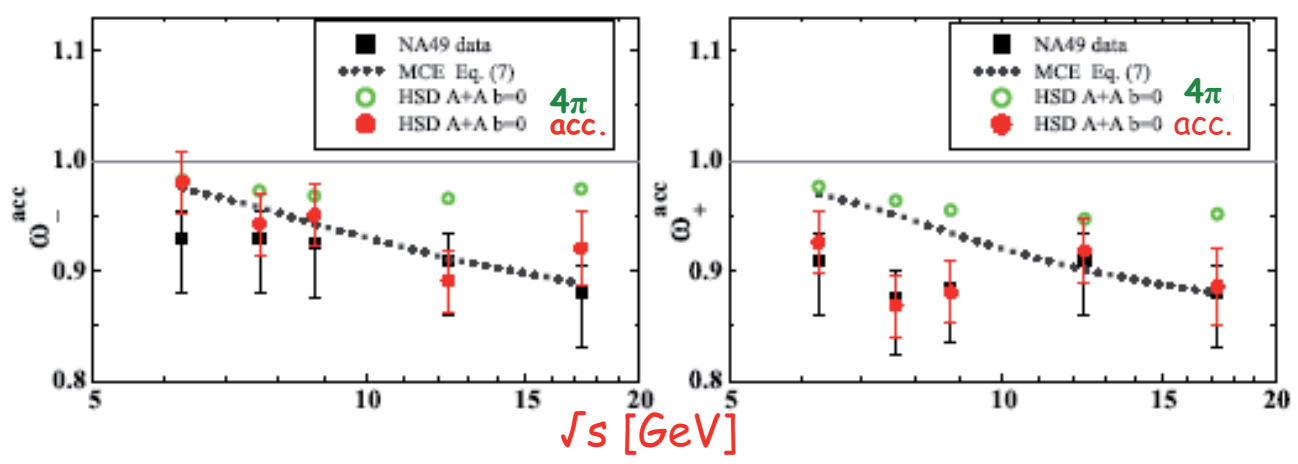

Strong dependence on acceptance

V.Konchakovski et al., nucl-th/0703052

\section{Event-by-event fluctuations, cont'd}

$\mathbf{p}_{\mathrm{T}}$ fluctuation $\sigma\left(p_{T}\right)_{d y n}=\sqrt{<\Delta p_{T i} \Delta p_{T j}>} / \overline{p_{T}}$

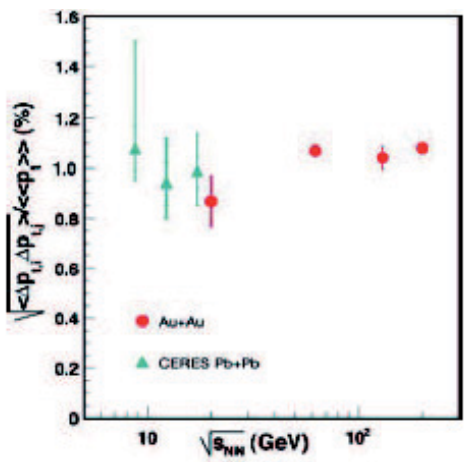

No hint at CEP phenomena C.Pruneau et. al., NP A774 (2006) 661
The relative deviation of the $K / \pi$ fluctuation width from the mixed event background width $\quad \sigma_{\text {dyn }}=\sqrt{\sigma_{\text {data }}^{2}+\sigma_{\text {mix }}^{2}}$

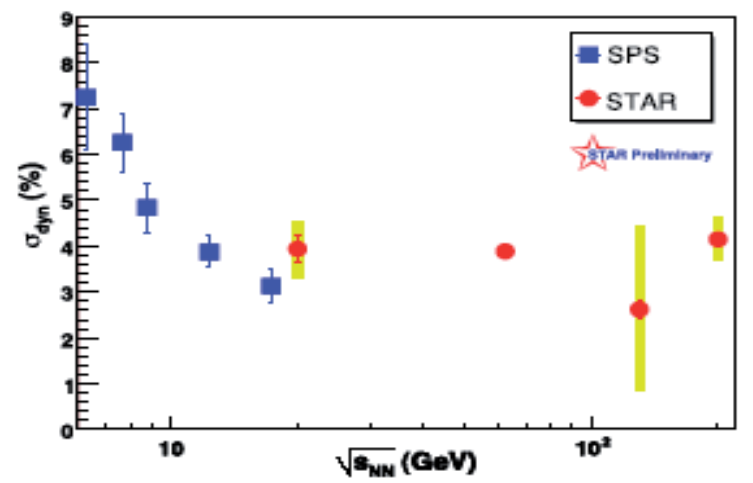

Exhibits a steep rise towards lower energies $\left(ل_{s}=6.2 \mathrm{GeV}\right)$

S.Das et al., SQM06 


\section{Hanbury-Brown and Twiss correlations of}

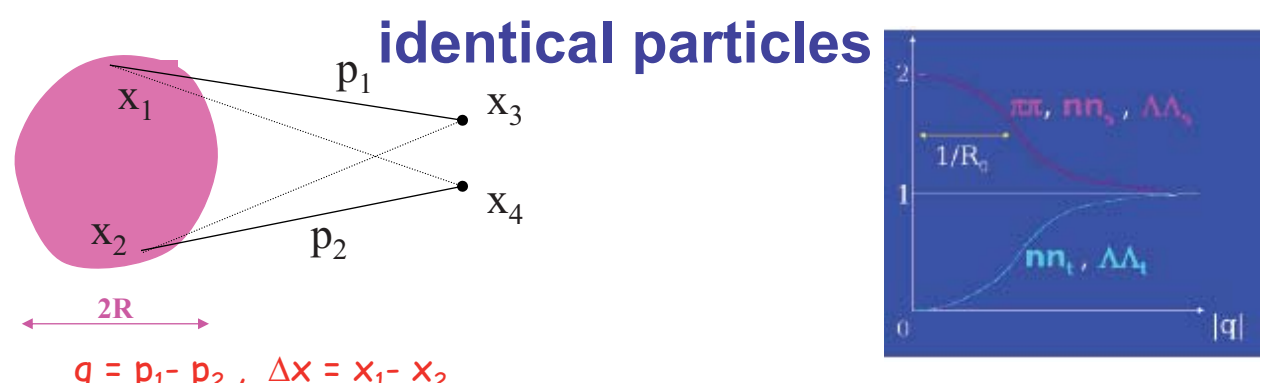

$q=p_{1}-p_{2}, \Delta x=x_{1}-x_{2}$

$\mathcal{A}_{12}=\frac{1}{\sqrt{2}}\left[e^{i p_{1} x_{1}+i p_{2} x_{2}}+(-1)^{s} e^{i p_{1} x_{2}+i p_{2} x_{1}}\right], s-$ spin of a pair

$\left|\mathcal{A}_{12}\right|^{2}=1+(-1)^{s} \cos \left[q\left(x_{1}-x_{2}\right)\right]$

$C_{2}(q)=\int d^{4} x_{1} d^{4} x_{2}\left|\mathcal{A}_{12}\right|^{2} \rho(x, y)$

Correlation function $\quad C_{2}=1+(-1)^{S}<\operatorname{Cos} q \Delta x>$

$\rightarrow 1+\lambda \exp \left(-R_{\text {long }}^{2} q_{\text {long }}^{2}-R_{\text {side }}^{2} q_{\text {side }}^{2}-R_{\text {out }}^{2} q_{\text {out }}^{2}-2 R_{\text {out }}^{2} q_{\text {out }} q_{\text {long }}\right)$

\section{HBT correlations: experimental status of $\left(\pi^{-} \pi^{-}\right)$}

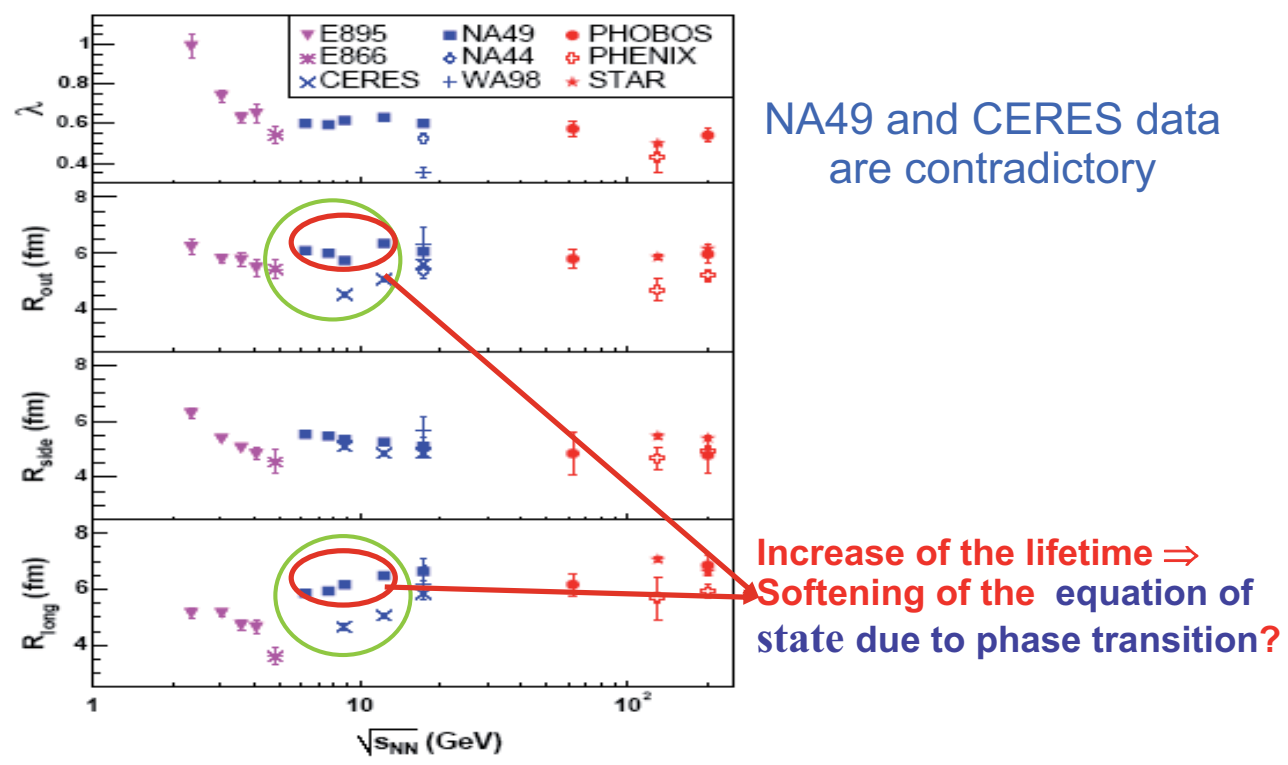




\section{Directed flow $v_{1}$ \& elliptic flow $v_{2}$}

Non central Au+Au collisions :

interaction between constituents leads to a pressure gradient $=>$ spatial asymmetry is converted to an asymmetry in momentum space $=>$ collective flow

$\frac{d N}{\operatorname{dyp}_{\mathrm{T}} d p_{\mathrm{T}} \mathrm{d} \varphi}=\frac{\mathrm{dN}}{\mathrm{dyp}_{\mathrm{T}} \mathrm{dp}} \frac{1}{2 \pi}\left(1+2 \mathrm{v}_{1} \cos (\varphi)+2 \mathrm{v}_{2} \cos (2 \varphi)+\ldots\right)$

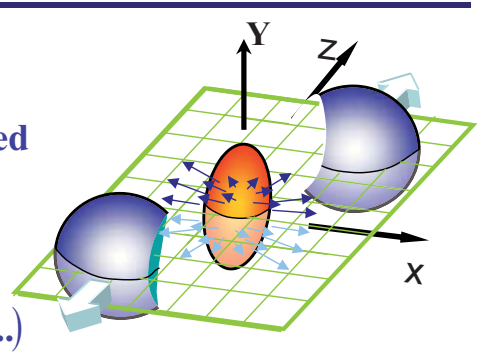

$\mathbf{v}_{1}=<\frac{\mathbf{p}_{\mathrm{x}}}{\mathbf{p}_{\mathrm{T}}}>-$ directed flow

$\mathbf{v}_{\mathbf{2}}=<\frac{\mathbf{p}_{\mathrm{x}}{ }^{2}-\mathbf{p}_{\mathbf{y}}{ }^{2}}{\mathbf{p}_{\mathbf{x}}{ }^{2}+\mathbf{p}_{\mathbf{y}}{ }^{2}}>-$ elliptic flow

$V_{2}>0$ indicates in-plane emission of particles

$\mathrm{V}_{2}<0$ corresponds to a squeeze-out perpendicular

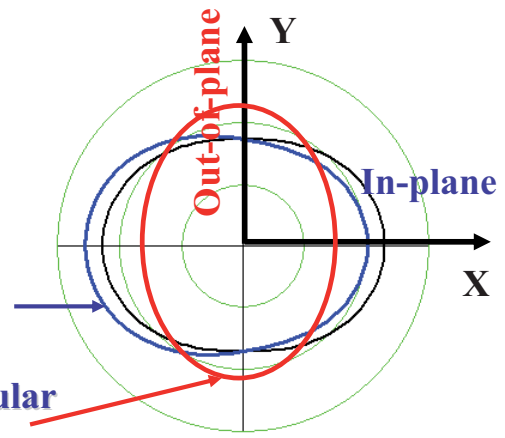
to the reaction plane (out-of-plane emission)

\section{Collective flow: $v_{2}$ excitation function}

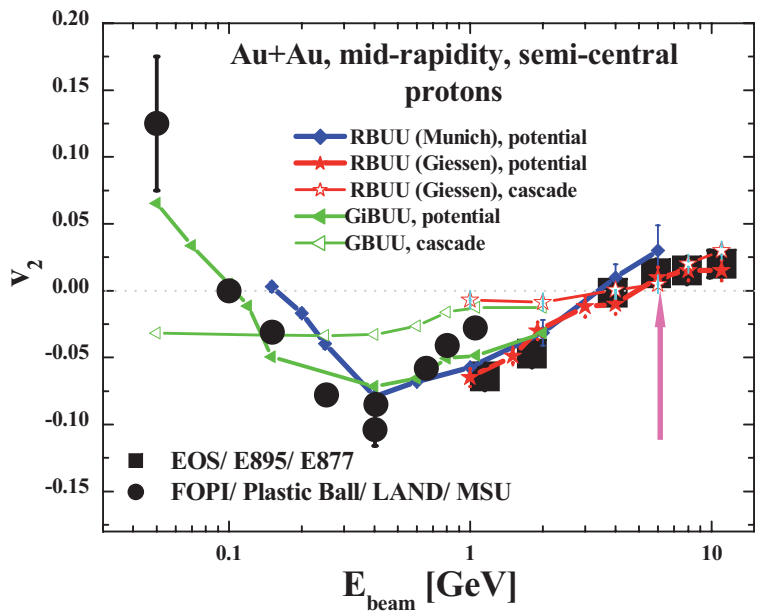

- Proton $v_{2}$ at low energy shows sensitivity to the nucleon potential.

- Cascade codes fail to describe the exp. data.

- AGS energies: transition from squeeze-out to in-plane elliptic flow 


\section{$v_{1}$ and $v_{2}$ flows for $\mathrm{Pb}+\mathrm{Pb}$ at $40 \mathrm{AGeV}$}
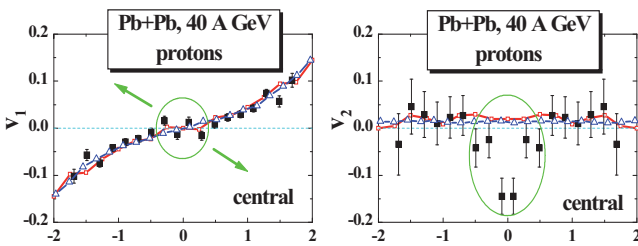

Small wiggle in $v_{1}$ at midrapidity is not described by HSD and UrQMD

Too large elliptic flow $v_{2}$ at midrapidity from HSD and
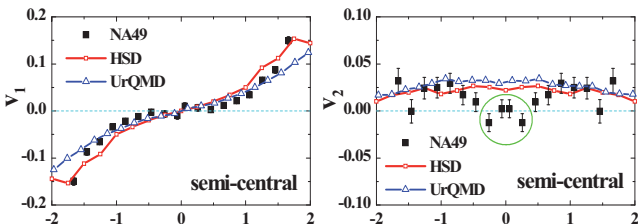
UrQMD for all centralities !

Experiment (NA49): breakdown of elliptic $v_{2}$ flow at midrapidity !
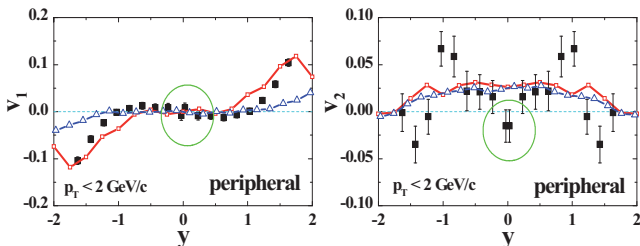<smiles>C1=CCCC1</smiles>

\section{Signature for a first} order phase transition

H.Stoecker et al., JPG 31 (2005) S929

\section{3 fluid hydro with hadronic EoS}
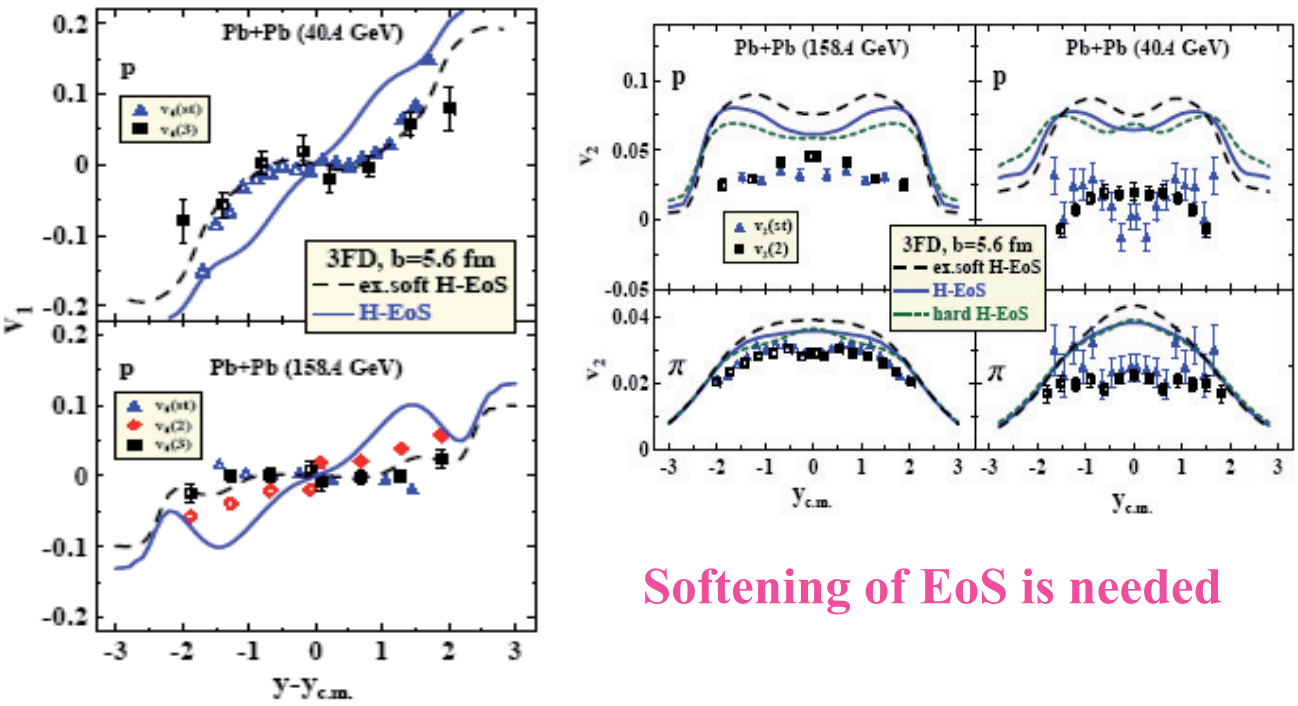

Softening of EoS is needed

Y.Ivanov, V.Russkikh, arXiv:0710.3708 
Flow scaling at partonic level

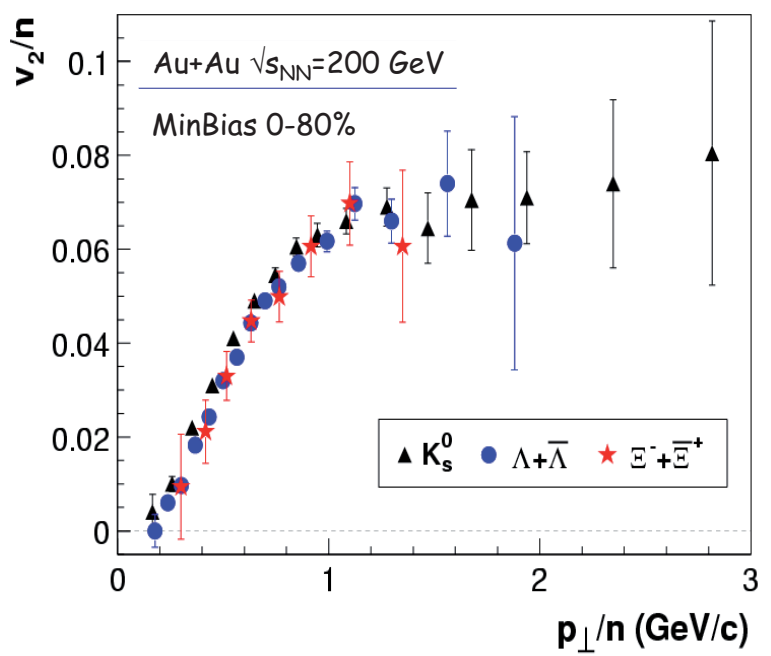

- The complex behaviour of $v_{2}$ can be « simply » explained at partonic level

$$
\begin{aligned}
& v_{2}^{p}\left(p_{\mathrm{t}}\right)=\frac{v_{2}^{B}\left(3 p_{\mathrm{t}}\right)}{3} \\
& v_{2}^{p}\left(p_{\mathrm{t}}\right)=\frac{v_{2}^{M}\left(2 p_{\mathrm{t}}\right)}{2} \\
& v_{2}^{p}\left(p_{\mathrm{t}}\right)=\frac{v_{2}^{h}\left(n p_{\mathrm{t}}\right)}{n}
\end{aligned}
$$

at intermediate $p_{\mathrm{T}}$ !

Idea of flow per constituent - Coalescence/Recombination At RHIC energies elliptic flow develops at partonic level. What about $40 \mathrm{AGeV}$ ?

\section{Chiral condensate and dileptons}

$<\bar{q} q>_{0}=(-230 \mathrm{MeV})^{3}$

$$
m_{V}^{2} \sim<\bar{q} q>\text { (G.Brown, M.Rho) }
$$

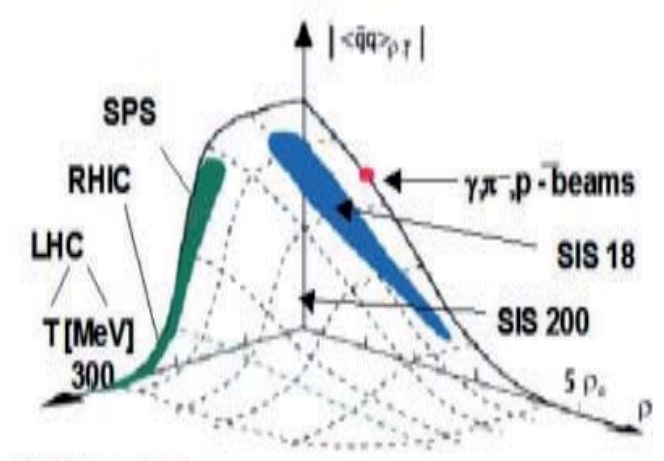

W. Weise et al.

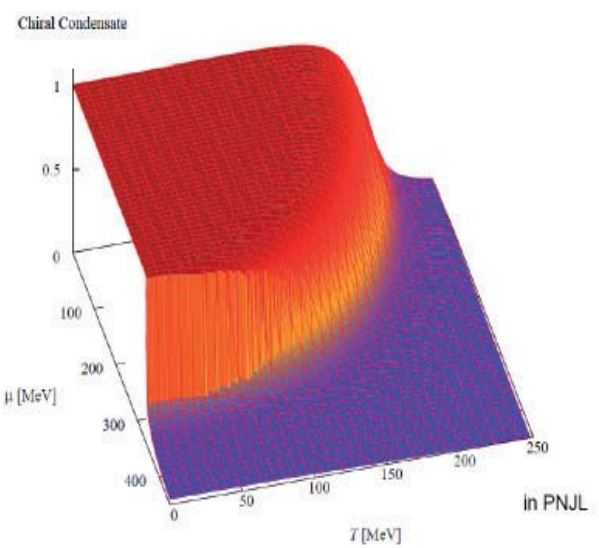

KFukushima, QM2008 


\section{Charactrerstics of dilepton experiments}
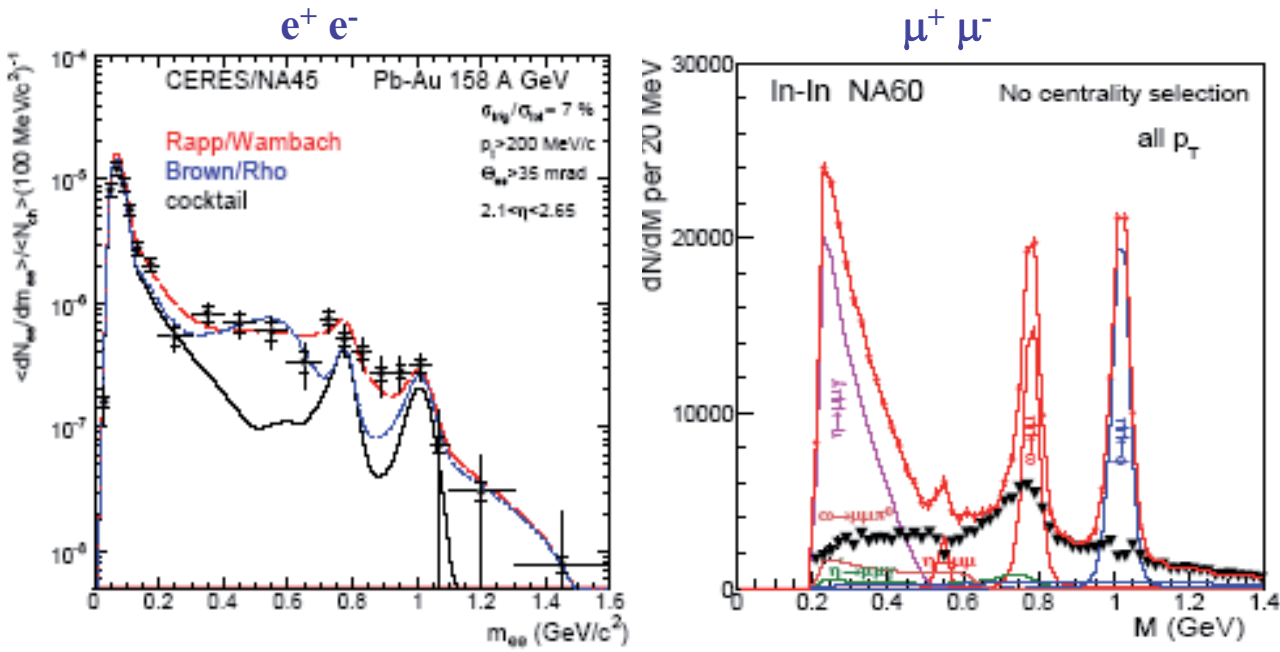

HADES: $\mathrm{C}+\mathrm{C} \longrightarrow \mathrm{e}^{+} \mathrm{e}^{-}+\mathrm{X}$ at $1 \& 2 \mathrm{~A} \mathrm{GeV}$

\section{Dilepton production}

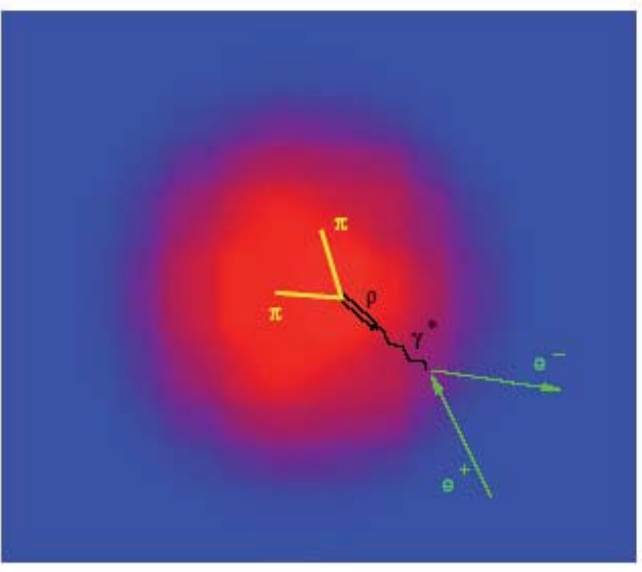

In-medium effect

J.Brown and M.Rho, Phys.

Rev. Lett. 66 (1991) 2720;

Phys. Rept. 269 (1996) 333

R.Rapp and J.Wambach, Adv. Nucl. Phys. 25 (2000) 1

$$
\begin{array}{r}
\frac{d^{4} N_{e e}}{d q^{4}}=-\int d x^{4} \mathcal{L}(M) \frac{\alpha^{2}}{\pi^{3} q^{2}} \frac{\operatorname{Im}_{e m}\left(q, T(x), \mu_{B}(x)\right)}{e^{q_{0} / T(x)}-1} \\
q^{2}=M^{2}=q_{0}^{2}-\vec{q}^{2}
\end{array}
$$




\section{$\rho$-meson spectral function}

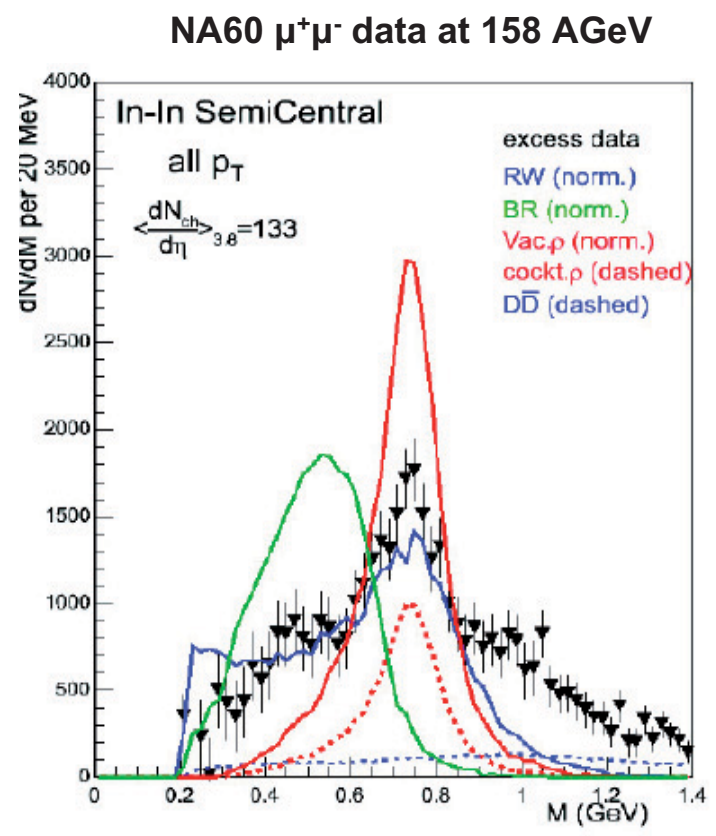

High precision NA60 data allow to distinguish among in-medium models

Clear evidence for a broadening of the $\rho$-meson spectral function (chiral symmetry restoration?)

NA60 Collaboration, PRL 96 (2006) 162302

\section{Dropping $\rho$-mass scenarios}

In the one-loop approximation

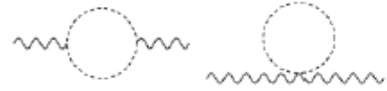

electromagnetic current correlation function $\operatorname{Im} \Pi_{e m}(M)=\frac{m_{\rho}^{4}}{g^{2}} \frac{I m \Pi}{\left(M^{2}-m_{\rho}^{2}\right)^{2}+(\operatorname{Im} \Pi)^{2}}$ $\rho$-meson self-energy for free pions

$$
\begin{gathered}
I m \Pi=-\frac{g_{\rho \pi \pi}^{2}}{48 \pi} \frac{\left(M^{2}-4 m_{\pi}^{2}\right)^{3 / 2}}{M} \\
g_{\rho \pi \pi}=6.05 g=5.03 m_{\rho}=770 \mathrm{MeV} \\
m_{\rho} \rightarrow m_{\rho}^{\star}, \quad \frac{m_{\rho}^{2}}{g}=\frac{m_{\rho}^{\star 2}}{g^{\star}} \\
m_{\rho}^{*}=m_{\rho}\left(1-0.15 \frac{n_{B}(x)}{n_{0}}\right)\left\{1-\left[\frac{T(x)}{T_{c}}\right]^{2}\right\}^{0.3}
\end{gathered}
$$

T.Hatsuda, S.H. Lee, PR C46 (1992) R34
V.Skokov and V.Toneev, Phys. Rev. C73 (2006) 021902

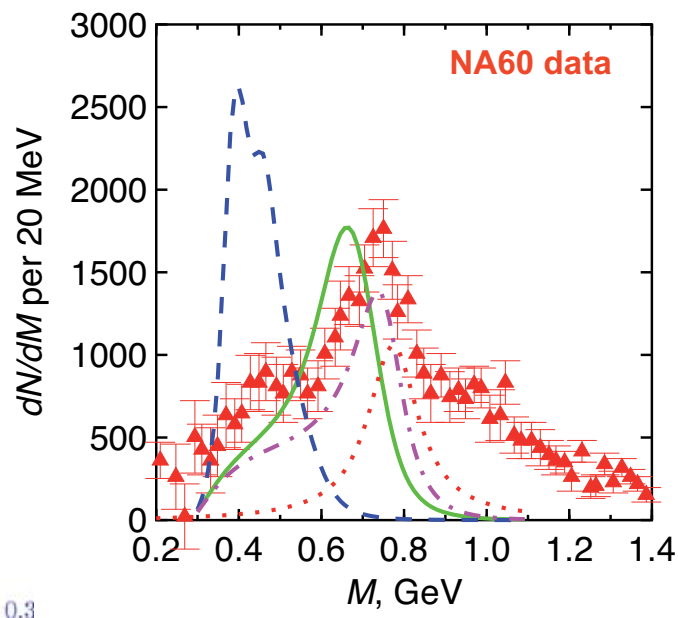

Dashed-dotted: no in-medium effect Dotted: $\rho$-mesons from freeze-out 


\section{Remarks}

Brown-Rho scaling $\frac{m_{\rho}^{\star}}{m_{\rho}}=\left(\frac{<\bar{q} q>^{\star}}{<\bar{q} q>}\right)^{1 / 2}$

To be consistent with QCD sum rules, both the collision broadening and dropping $\rho$-mass should be taken into account (relation to the quark condensate ?)

QCD sum rules
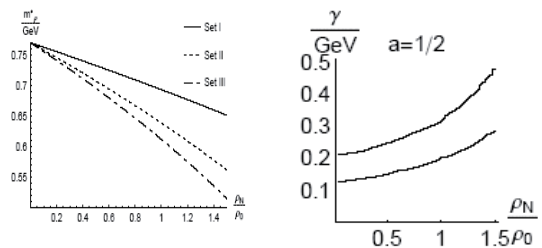

J.Ruppert, T.Renk and B.Muller, Phys. Rev. C73 (2006) 034907

Dileptons carry a direct information on the $\rho$-meson spectral function only if the assumption on vector dominance is valid (G.E.Brown and M.Rho, nuclth/0509001; nucl-th/0509002). This is not so in the Harada- Yamawaki vector manifestation of Hidden Local Symmetry - HLS (M.Harada and K.Yamawaki, Phys. Rept. 381 (2003) 1). In this case the electromagnetic coupling is governed by the parameter a of HLS theory which runs with temperature and density (see G.E. Brown et al., arXiv:0804.3196).

Experimental subtraction of the hadronic cocktail: what about in-medium $\omega$-mesons? $\rho-\omega$ interference?

\section{Q Quark(anti-quark)-hadron bremsstrahlung}

In the soft photon approximation

(similarly to the pn bremsstrahlung)

$$
\begin{gathered}
\frac{d \sigma_{q N}^{l^{+} l^{-}}(s, M)}{d M^{2}}=\frac{\alpha^{2}}{3 \pi^{2}} \frac{\bar{\sigma}(s)}{M^{2}} \ln \left[\frac{s^{1 / 2}-m_{N}-m_{\bar{q}}}{M}\right] \times \mathbf{K} \quad l^{-} \uparrow \\
\bar{\sigma}(s) \approx 2 \sigma_{e l}^{q N}\left[\frac{s}{\left(m_{N}+m_{\bar{q}}\right)^{2}}-1\right] \quad \bar{\sigma}_{e l}^{q N}=\left[\frac{18(m b \cdot G e V) m_{N}}{s-\left(m_{N}+m_{\bar{q}}\right)^{2}}+10 m b\right] \times \frac{\mathbf{1}}{\mathbf{3}}
\end{gathered}
$$

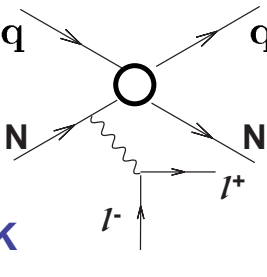

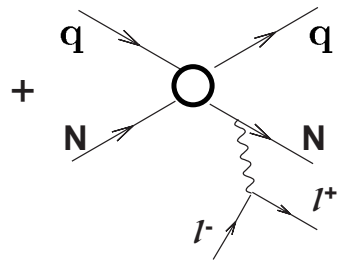

Dilepton emission rate

$$
\frac{d R_{\bar{q} N}^{l^{+} l^{-}}}{d M^{2}}=\int \frac{d^{3} k_{\bar{q}}}{(2 \pi)^{3}} f\left(\mathbf{k}_{\bar{q} N}\right) \int \frac{d^{3} k_{N}}{(2 \pi)^{3}} f\left(\mathbf{k}_{N}\right) \frac{d \sigma_{\bar{q} N}^{l^{+} l^{-}}(s, M)}{d M^{2}} v_{r e l}
$$

(3) Intermadiate mass dileptons. Quark-antiquark annihilation from the plasma phase (K.Dusling and I.Zahed, arXiv:0712.1982; hep-ph/0701253) were considered but not from the q-h mixed phase ! 
First estimate of the $q-h$ bremsstrahlung (preliminary)

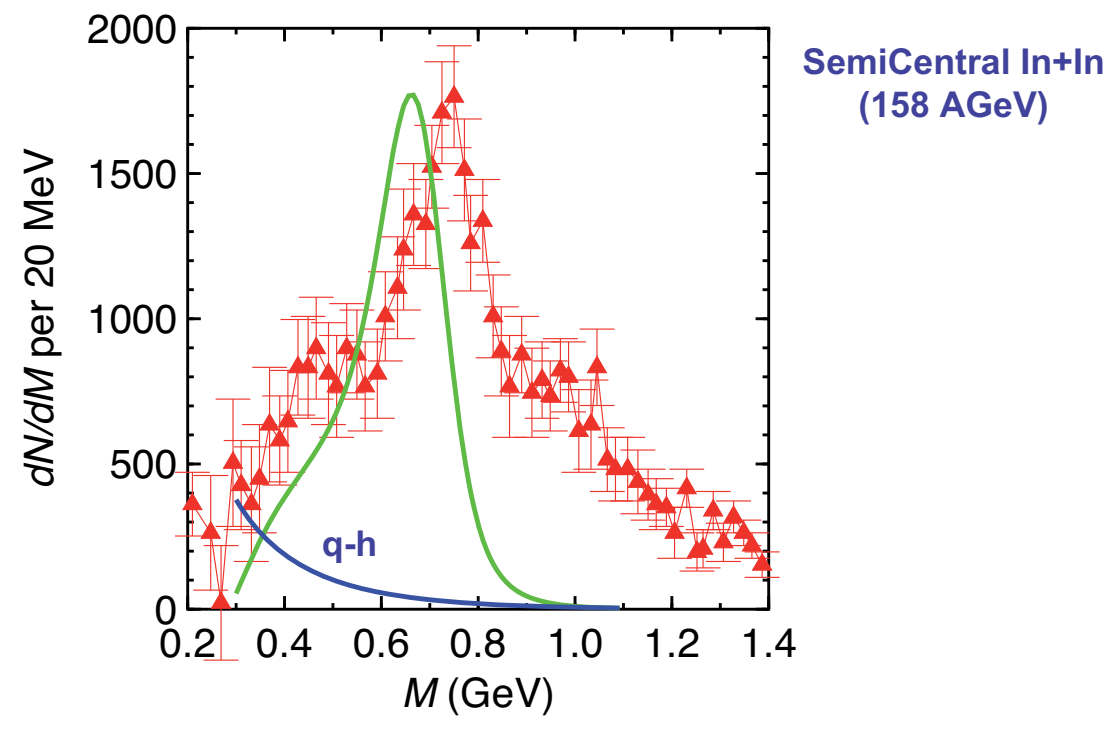

$\mathrm{K}=10 \quad$ Further study is needed

\section{Charmonium in heavy-ion collisions}

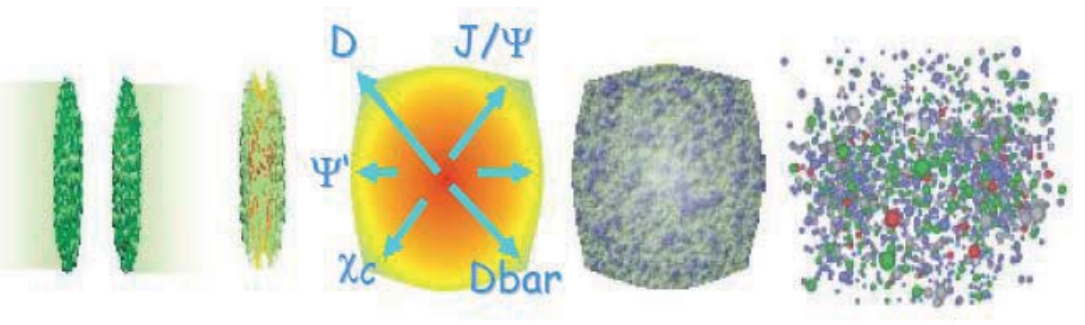

Charmonium creation versus absorption

'hidden' and 'open' charm will be discussed in other lectures 


\section{Production of two correlated photons}

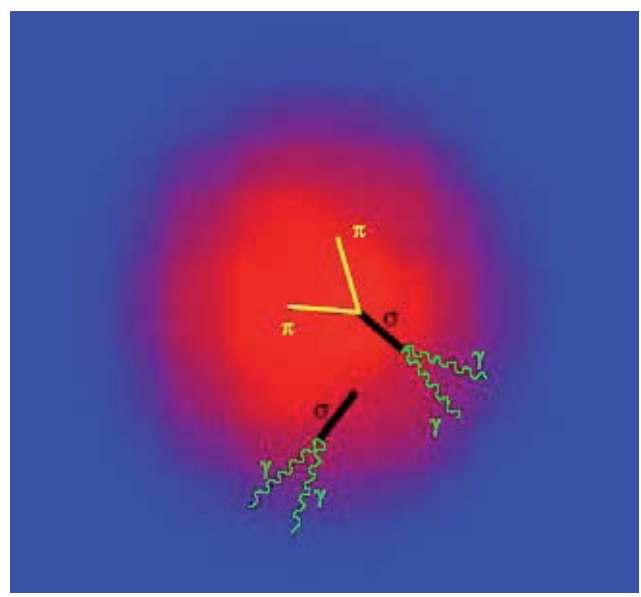

\section{A sign of chiral symmetry restoration}

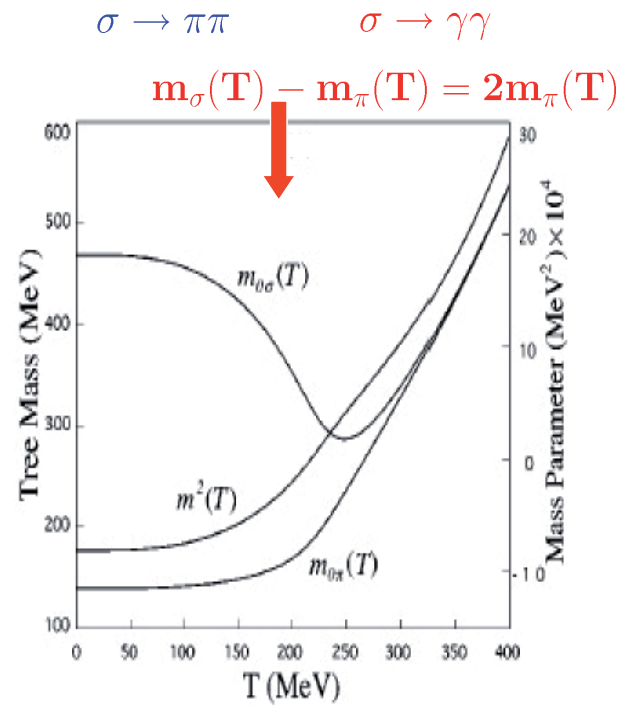

S.Chiki, T.Hatsuda, PR D58 (1998) 076001
Invariant mass distribution for $\sigma \rightarrow \gamma \gamma$

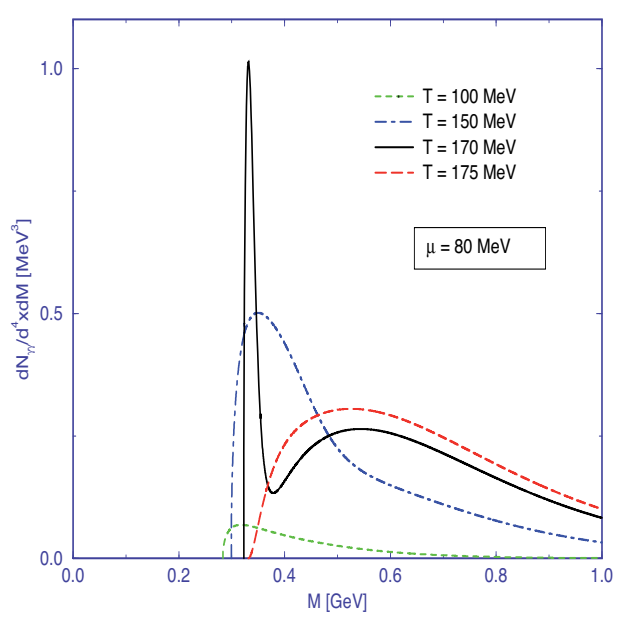

M.Volkov et al., PL B424 (1998) 235 


\section{$\pi^{+} \pi^{-}=>\gamma \gamma$ in NJL model}
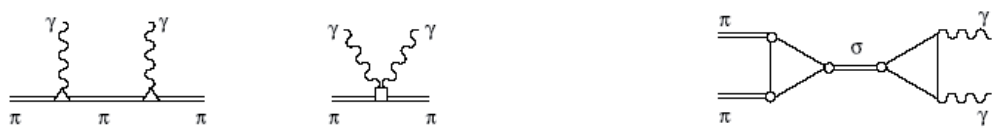

$$
\sigma^{\pi^{+} \pi^{-} \rightarrow \gamma \gamma}(s)=\sigma_{\text {Born }}(s)+\sigma_{\text {interf }}(s)+\sigma_{\text {res }}(s)
$$

$$
\begin{aligned}
\sigma_{\text {Born }}(s) & =16 \sigma_{0}\left(2-\kappa^{2}-\frac{1-\kappa^{4}}{2 \kappa} \ln \left[\frac{1+\kappa}{1-\kappa}\right]\right) \\
\sigma_{\text {interf }}(s) & =4 \sigma_{0} s \operatorname{Re}\left[\mathcal{A}_{\pi^{+} \pi^{-} \rightarrow \gamma \gamma}(s)\right] \frac{1-\kappa^{2}}{\kappa} \ln \left[\frac{1+\kappa}{1-\kappa}\right] \\
\sigma_{\text {res }}(s) & =\sigma_{0} s^{2}\left|\mathcal{A}_{\pi^{+} \pi^{-} \rightarrow \gamma \gamma}(s)\right|^{2}
\end{aligned}
$$

where $\sigma_{0}=\pi \alpha^{2} / 4 s \kappa, \kappa^{2}=1-4 M_{\pi}^{2}(T, \mu) / s$, and

$$
\begin{aligned}
\mathcal{A}_{\pi^{+} \pi^{-} \rightarrow \gamma \gamma} & =\frac{1}{\left(6 \pi f_{\pi}(T, \mu)\right)^{2}}\left[\frac{40 m(T, \mu)}{M^{2}(T, \mu)-s-i \sqrt{s} \Gamma_{\sigma}(s \mid T, \mu)}\right. \\
& \left.\times f_{1}(\mu, T)-f_{2}(\mu, T)\right]
\end{aligned}
$$

V.Yudichev, Round Table I, Dubna, 2005

The gap equation

$$
m(T, \mu)-m_{0}=8 m(T, \mu) G I_{1}^{\wedge}(m \mid T, \mu)
$$

$$
I_{1}^{\wedge}(m \mid T, \mu)=\frac{N_{c}}{4 \pi^{2}} \int^{\wedge} \frac{k^{2}}{E(k)}(1-n(k ; T, \mu)-\bar{n}(k ; T, \mu)) d k
$$

\begin{tabular}{c|c|c|c}
\hline$T=\mu=0$ & $\mathrm{G}\left[\mathrm{GeV}^{-2}\right]$ & $\wedge[\mathrm{MeV}]$ & $m_{0}[\mathrm{MeV}]$ \\
\hline Type I, $M_{\pi}, f_{\pi},<\psi \psi>$ & 11.7205 & 618.7 & 5.76 \\
Type II, $M_{\pi}, f_{\pi}, \rho \rightarrow \pi \pi$ & 3.4105 & 1037.4 & 2.08 \\
\hline
\end{tabular}
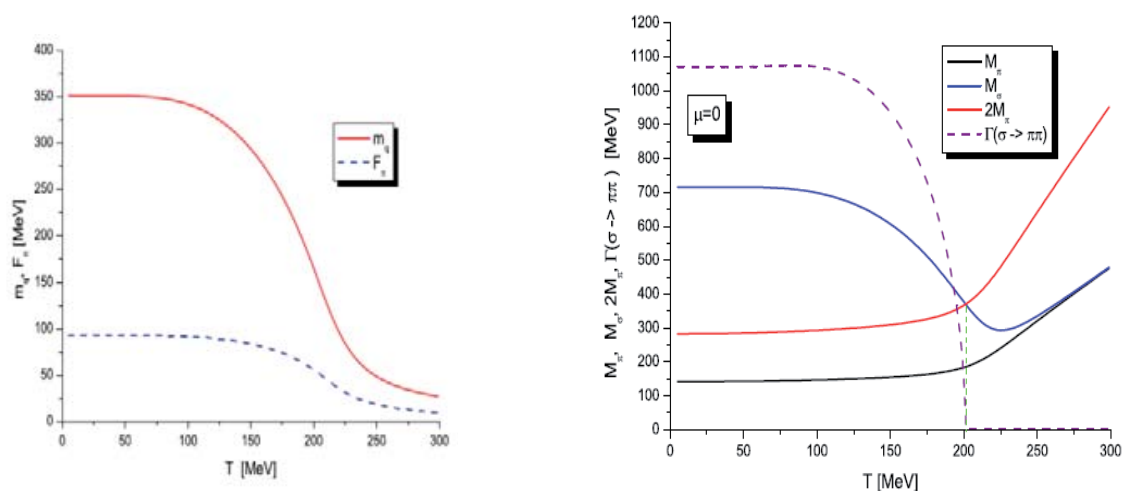


\section{Pion annihilation into 2 photons (preliminary)}

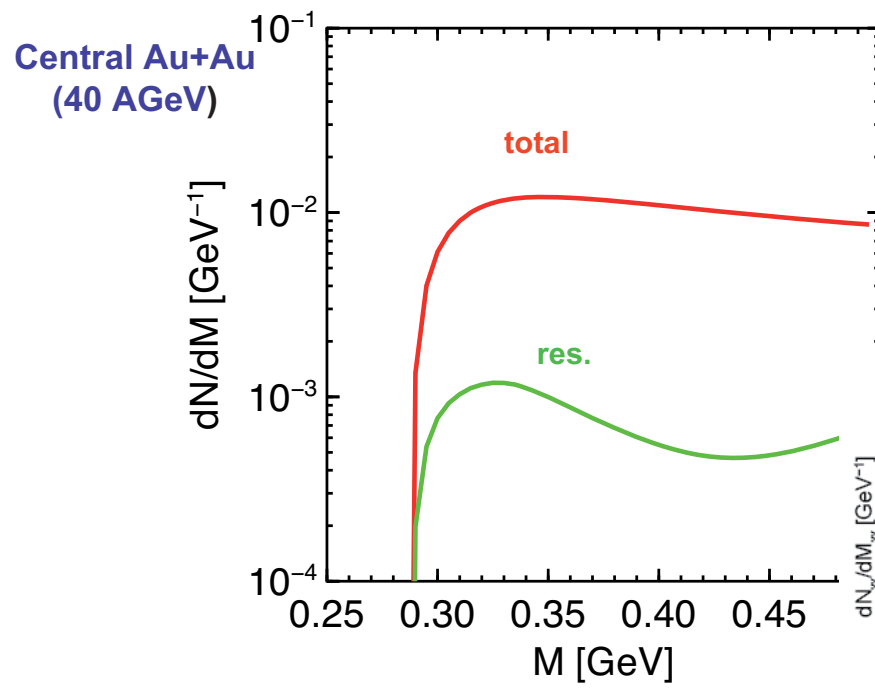

Maximum is noticeably washed out but the yield is not so small

V.Toneev and V.Skokov, Round Table I, Dubna (2005)

\section{Locating the QCD critical point: theoretical predictions}

Models, lattice

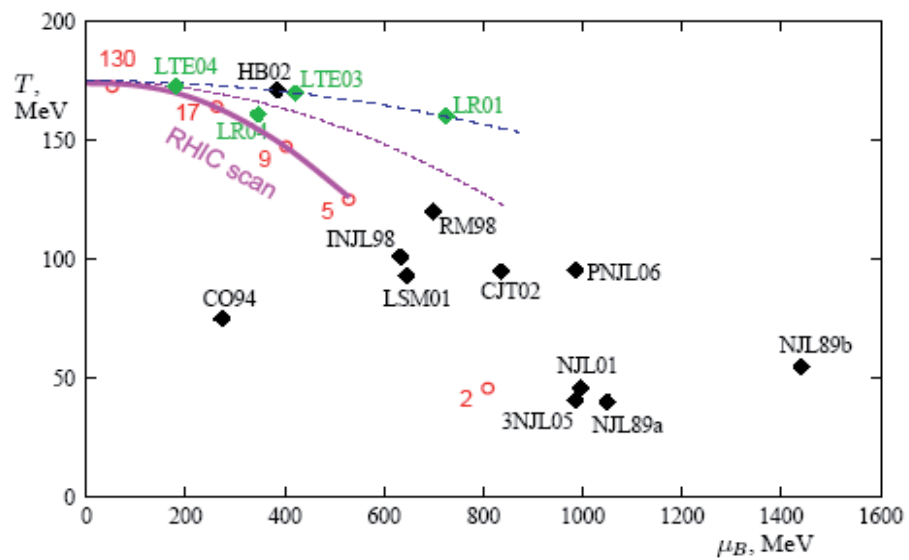

Freezeout points from thermal model fits to SIS/AGS/SPS/RHIC data (Braun-Munzinger, Redlich, Stachel) 


\section{Critical point on the lattice}
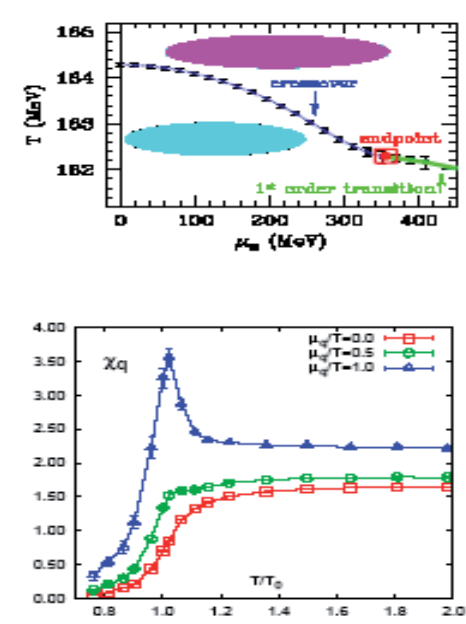

There is a peak in $X_{q}$ but not in $X_{1}$
Several approaches:

( Reweighting: Fodor-Katz

2 2001: $\mu_{B} \sim 725 \mathrm{MeV}$

2 2004: $\mu_{B} \sim 360 \mathrm{MeV}$ (smaller $m_{q}$ and larger $V$ )

2. Taylor expansion: Bielefeld-Swansea (to $\mu^{6}$ )

f 2003: $\mu_{B} \sim 420 \mathrm{MeV}$

- 2005: $300 \mathrm{MeV} \lesssim \mu_{B} \lesssim 500 \mathrm{MeV}$

- Taylor expansion: Gavai-Gupta (to $\mu^{8}$ )

2 From convergence radius: $\mu_{B} \sim 180 \mathrm{MeV}$ (more precisely > $180 \mathrm{MeV}$ )

(Imaginary $\mu$ : deForcrand-Philipsen, Lombardo, et al

2. Sensitive to $m_{s}$, perhaps $\mu_{B} \gg 300 \mathrm{MeV}$

- Fixed density: deForcrand, Kratochvila; Density of states: Fodor, Katz, Schmidt.

? ? $\left(N_{f}=4\right.$, small volumes $)$

\section{Viscosity-to-entropy ratio}

minimum bias $\mathrm{Au}+\mathrm{Au}, \sqrt{\mathrm{s}}=\mathbf{2 0 0} \mathrm{GeV}$

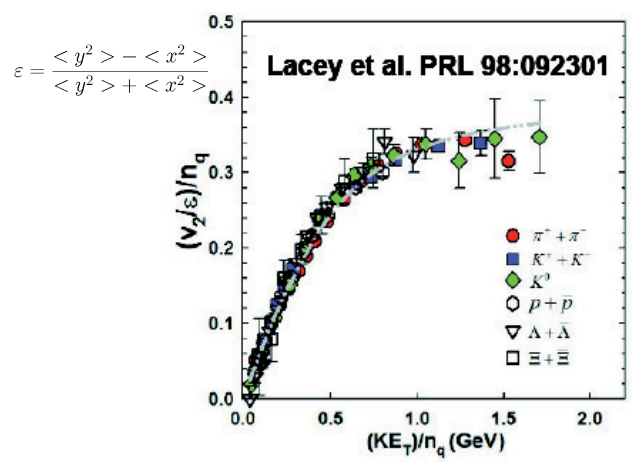

Hydrodynamic scaling

$T^{i j}=\delta^{i j} P-\eta\left(\partial^{i} u^{j}+\partial^{j} u^{i}-\frac{2}{3} \delta^{i j} \partial_{l} u^{l}\right)-\zeta \delta^{i j} \partial_{l} u^{l}$ Partonic fluid $\quad \frac{\eta}{s} \sim T \lambda_{f} c_{s}, \quad \sim 1.3 \times\left(\frac{1}{4 \pi}\right)$

Lower bound of $\eta / s=1 / 4 \pi$ in the strong coupling limit (P.Kovtun et al. PRL 94 (2005) 111601) $\eta / s$ for several substances

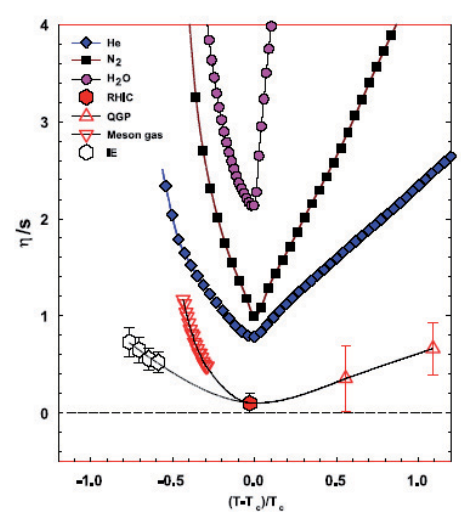

Strong indication for a minimum in the vicinity of $T_{c}$

L.P.Csernai et al. PRL 97 (2006) 152303; R.Lacey at al. PRL 98 (2007) 092301 


\section{Location of the CEP (?)}

Results for an isobar at the critical pressure $P_{c}$ and one above/below it

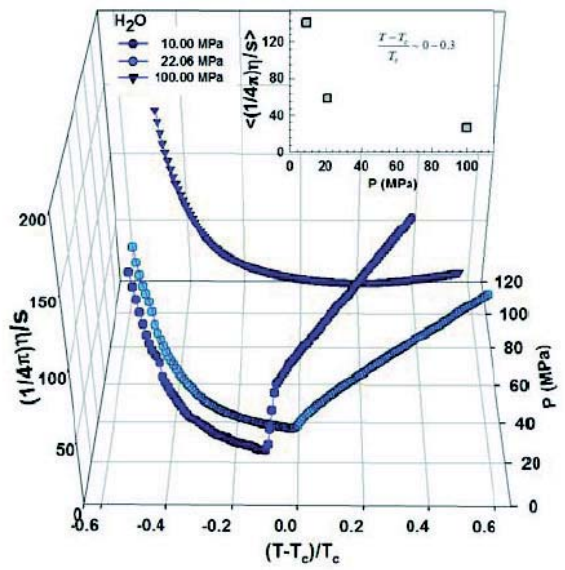

Flow excitation function

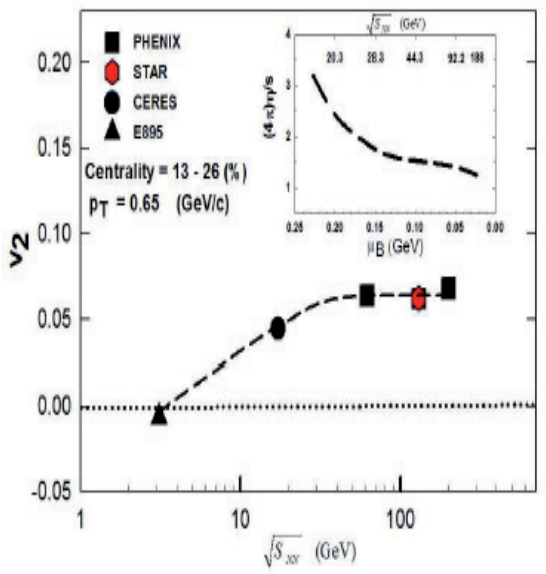

$\eta / s$ is a potential signal of the CEP

$\mathrm{T}-\mu_{\mathrm{B}}$ correlates at the freeze-out. First esatimate
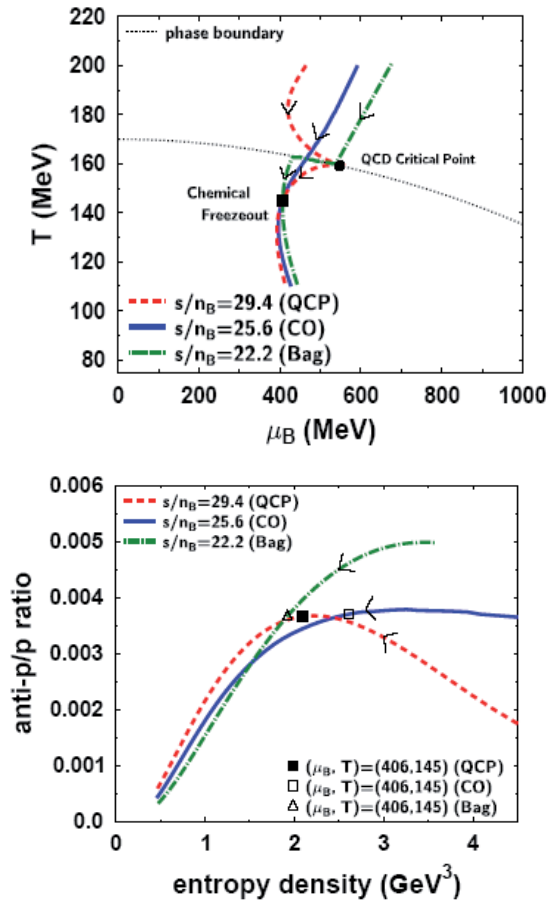

\section{Transverse rapidity dependence of the proton- antiproton ratio}

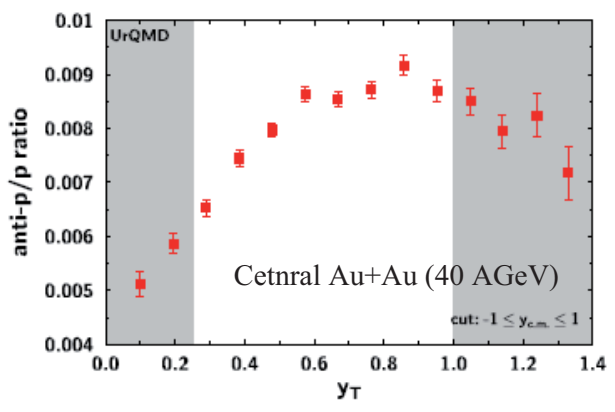

An unusual $\mathrm{y}_{\mathrm{T}}$ dependence of the p/anti-p ratio in a narrow beam energy window would signal the presence of the CEP

Asakawa et al., arXiv:0803.2449 


\section{Concluding remarks}

- PT in HIC is not stable state but transient one

- PT evolves in finite space/time

- after PT the system continues to evolve

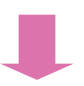

- There is not a single decisive (crucial) signal

- Every signal is essentially washed out due to subsequent interactions

\section{Scanning of all signals in energy and impact parameter}

